Draft VERSion February 27, 2019

Typeset using $\mathrm{LTT}_{\mathrm{E}} \mathrm{X}$ preprint2 style in AASTeX62

\title{
THE AXIAL ROTATION AND VARIABLE MACROTURBULENCE OF RR LYRAE AND RED HORIZONTAL BRANCH STARS
}

\author{
George W. Preston, ${ }^{1}$ Christopher Sneden, ${ }^{2}$ Merieme Chadid, ${ }^{3}$ Ian B. Thompson, ${ }^{1}$ And \\ Stephen A. SheCtman ${ }^{1}$ \\ ${ }^{1}$ Carnegie Observatories, 813 Santa Barbara Street, Pasadena, CA 91101, USA; gwp,ian,shec@obs.carnegiescience.edu \\ ${ }^{2}$ Department of Astronomy and McDonald Observatory, The University of Texas, Austin, TX 78712, USA; \\ chris@verdi.as.utexas.edu \\ ${ }^{3}$ University of Côte d'Azur, Nice Sophia-Antipolis University, ARTEMIS-UMR 7250, CS 34229, 06304 Nice, France; \\ Merieme.Chadid@unice.fr
}

\begin{abstract}
We have derived relations between full-width-half-maxima and equivalent widths of metallic absorption lines in the spectra of RR Lyrae stars to estimate new upper limits on the axial equatorial rotational velocities of RR Lyrae and metal-poor red horizontal branch stars (RHB). We also have derived the variations of RR Lyrae macroturbulent velocities during the pulsation cycles. In RRab cycles the line widths are dominated by phase-dependent convolutions of axial rotation and macroturbulence, which we designate as $V_{\text {macrot }}$. The behavior of $V_{\text {macrot }}$ is remarkably uniform among the RRab stars, but the behavior of $V_{\text {macrot }}$ among RRc stars varies strongly from star to star. The RRab stars exhibit an upper limit on $V_{\text {macrot }}$ of $5 \pm 1 \mathrm{~km} \mathrm{~s}^{-1}$ with weak evidence of an anti-correlation with $T_{\text {eff }}$. The RRc minima range from 2 to $12 \mathrm{~km} \mathrm{~s}^{-1}$. The abrupt decline in large rotations with decreasing $T_{\text {eff }}$ at the blue boundary of the instability strip and the apparently smooth continuous variation among the RRab and RHB stars suggests that HB stars gain/lose surface angular momentum on time scales short compared to HB lifetimes. $V_{\text {macrot }}$ values for our metal-poor RHB stars agree well with those derived by Fourier analysis of an independent but less metal-poor sample of Carney et al. (2008); they conform qualitatively to the expectations of Tanner et al. (2013). A general conclusion of our investigation is that surface angular momentum as measured by $V_{\text {rot }} \sin (i)$ is not a reliable indicator of total stellar angular momentum anywhere along the HB.
\end{abstract}

Keywords: methods: observational techniques: spectroscopic - stars: atmospheres stars: oscillations stars: variables: RR Lyr

\section{INTRODUCTION}

Variations of turbulence during the pulsation cycles of classical Cepheids (Breitfellner \& Gillet 1993, Stift \& Gillet 1994, Bersier \& Burki 1996) and RR Lyrae stars (Chadid \& Gillet 1996, Fokin et al. 1999) have been well- documented. Individual studies have different definitions of turbulence and procedures for its extraction from stellar line profiles. The disentanglement of stellar line broadening due to axial rotation from turbulence, however defined, is difficult in all of these investigations, which mainly have been concerned with the genera- 


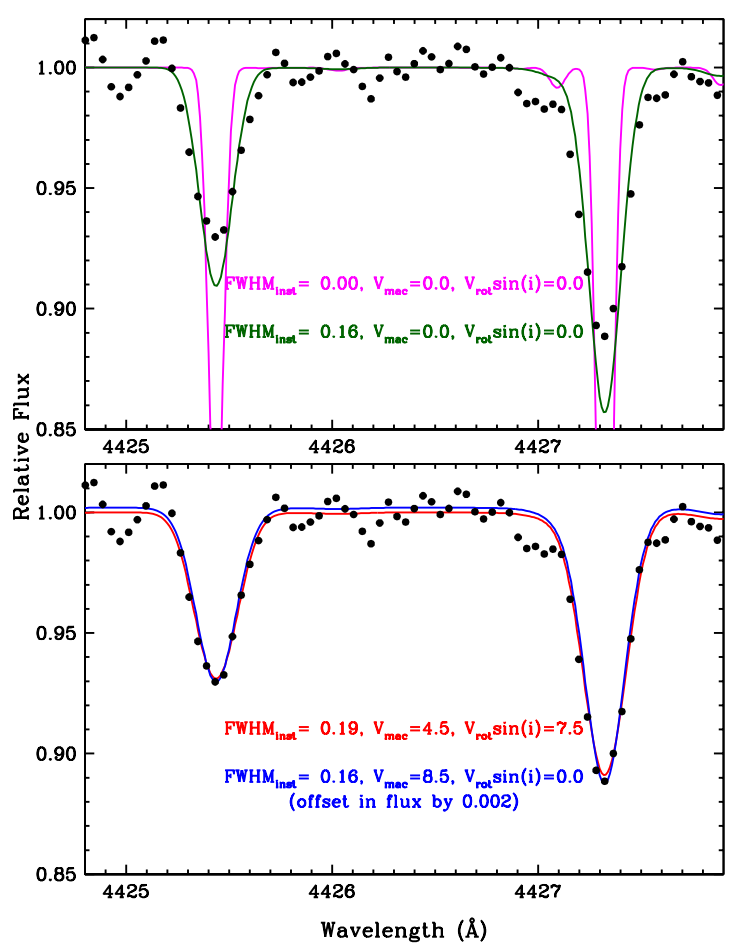

Figure 1. Top panel: Observed and synthetic profiles of Ca I $4425.44 \AA$ and Fe I $4427.32 \AA$, two unsaturated lines in the spectrum of RRab X Ari. The black dots represent the observed spectrum. The magenta line is the raw synthetic spectrum, broadened only by thermal and microturbulent velocities assumed in the spectrum computations. The dark green line shows the raw spectrum broadened by the spectrograph slit function only. Bottom panel: The same observed spectrum is compared to two synthetic spectra. Each of these has the same thermal, microturbulent, and spectrograph slit broadening, but the red line has $V_{m a c}=4.0 \mathrm{~km} \mathrm{~s}^{-1}$ and $V_{\text {rot }} \sin (i)=6.0 \mathrm{~km} \mathrm{~s}^{-1}$, and the blue line has $V_{\text {mac }}=7.5 \mathrm{~km} \mathrm{~s}^{-1}$ but no rotational broadening. A small vertical shift has been added to the blue line for plotting clarity.

tion of turbulence and its effect on pulsating atmospheres.

This problem of disentanglement is illustrated in Figure 1, which shows observed and synthetic spectra of the $\mathrm{X}$ Ari, a very metal-poor $(\mathrm{Fe} / \mathrm{H}]=-2.6)$ RRab star, at phase $\phi=0.37$. In the top panel we show a computed spectrum (magenta color) of a small spectral region containing the Ca I $4425.44 \AA$ and Fe I $4427.32 \AA$ lines. This spectrum was generated with the synthetic spectrum software and model atmosphere parameters described by Chadid et al. (2017) and transition probabilities were adjusted to match the equivalent widths $(E W \mathrm{~s})$ of the two main lines. This computed spectrum only has line broadening that results from thermal and microturbulent velocity, and clearly does not match the observed spectrum (black dots). The dark green spectrum shows this synthesis with added Gaussian smoothing to account for the du Pont echelle spectrograph slit function $(R \equiv \lambda / \Delta \lambda \simeq 27,000$, or full width half maximum $F W H M \simeq 0.16 \AA$ at $4426 \AA$ ). This accounts for most but not all of the observed line profiles. The bottom panel shows two convolutions with identical $F W H M$ values but very different Maxwellian macroturbulent and rotational parameters (blue, $V_{m a c}=7.5 \mathrm{~km} \mathrm{~s}^{-1}$, $V_{\text {rot }} \sin (i)=0.0 \mathrm{~km} \mathrm{~s}^{-1}$; red, $V_{\text {mac }}=4.0 \mathrm{~km} \mathrm{~s}^{-1}$, $\left.V_{\text {rot }} \sin (i)=6.0 \mathrm{~km} \mathrm{~s}^{-1}\right)$. These profiles appear to be nearly identical in the wings. Because rotation remains virtually constant during a pulsation cycle, any variation of $F W H M$ with phase must be due to thermal and turbulent motions of gas elements within RRab atmospheres.

For 27 RRab stars Peterson et al. (1996) found a minimum $F W H M$ value near pulsation phase $\phi=0.35$, the approximate phase of maximum radius, derived from cross correlation of metal lines for radial velocity during the stellar pulsation cycles. They argued that the variation of $F W H M$ during pulsation cycles is due to variable turbulence and the minimum value is an unresolvable combination of turbulence and axial rotation. They derived an upper limit of $V_{\text {rot }} \sin (i)<10 \mathrm{~km} \mathrm{~s}^{-1}$ for the RR Lyrae stars, similar to that reported for classical Cepheids by Bersier \& Burki (1996). 
On the other hand, many blue horizontal branch (BHB) stars with $T_{\text {eff }}<11500 \mathrm{~K}^{1}$ are rapid rotators, some attaining $V_{\text {rot }} \sin (i) \sim 40 \mathrm{~km} \mathrm{~s}^{-1}$ (Peterson et al. 1995, Behr 2003a,b, Kinman et al. 2000, Recio-Blanco et al. 2004). Standard HB theory tells us that if these BHB rapid rotators evolve with constant Luminosity and constant angular momentum, then their rotation rates will go as $V_{\text {rot }} \sin (i) \propto T_{\text {eff }}^{2}$. In this case many of them will pass through the instability strip rotating more rapidly than $V_{\text {rot }} \sin (i) \sim 10 \mathrm{~km} \mathrm{~s}^{-1}$. None of them do so; hence the "Peterson Conundrum".

Here we address this puzzle by the study of additional samples of RRab and RRc RR Lyrae variables and red horizontal branch (RHB) stars. In $\S 2$ we describe the spectroscopic data sets and our extraction of observed broadening of metallic lines for each star in individual pulsation phases. In $\S 3$ and $\S 4$, for RRab and RRc stars, we derive $V_{\text {macrot }}$ quantities, which are combinaions of macro-turbulent and rotational broadening effects. We compare the $V_{\text {macrot }}$ values to values of micro-turbulent velocities $V_{m i c}$. In $\S 5$ we derive values of $V_{\text {macrot }}$ for very metalpoor RHB stars and compare them to values for RHB samples used in previous studies. Finally, we consider implications of the complex behavior of $V_{\text {macrot }}$ along the horizontal branch in $\S 6$.

\section{OBSERVATIONS AND PROCEDURES}

\subsection{The Observational Database}

1 As first noted by Grundahl et al. (1999) HB stars with $T_{\text {eff }} \lesssim 11,500 \mathrm{~K}$ have normal (approximately solar) $[\mathrm{X} / \mathrm{Fe}]$ values, while $\mathrm{HB}$ stars hotter than this value display abundance anomalies commonly associated with radiative levitation and gravitational settling (e.g., Michaud et al. 1983, Quievy et al. 2009, Théado et al. 2012). We adopt $11500 \mathrm{~K}$ as the upper temperature boundary for BHB stars to be included in our discussion.
The main observational data for this study were provided by several thousand echelle spectra of 35 RRab stars and 19 RRc stars acquired with the du Pont $2.5 \mathrm{~m}$ telescope of the Las Campanas Observatory in the years 2006-2014. ${ }^{2}$ Typical spectral resolving power is, as introduced above, $R \equiv \lambda / \Delta \lambda \simeq 27,000$ at $\lambda 5000 \AA$. Exposure times are limited to small fractions of the stellar pulsation periods, never exceeding $600 \mathrm{~s}(\simeq 0.01 P)$ and the typical signalto-noise ratio is $S / N \sim 15$ to 20 . The $S / N$ was improved by factors of two or more at all phases by co-addition of spectra in small phase intervals. Reduction procedures that resulted in wavelength-calibrated extractions of du Pont echelle spectra are described in detail by For et al. (2011a) and need not be repeated here. Basic data for the program stars are given in Table 1, where stars are listed in groups discussed in $\S 3-5$ below. For this paper we split the stellar sample into stars that are metal-poor (MP, $[\mathrm{Fe} / \mathrm{H}]<-1)$, and those that are metalrich $(\mathrm{MR},[\mathrm{Fe} / \mathrm{H}] \geq-1)$, consistent with the definition adopted by Chadid et al. (2017), Sneden et al. (2017, 2018). The mean temperatures for the RRab stars in this table are taken from Skarka (2014), and those for the RRc stars (which have only small $T_{\text {eff variations) are an as- }}$ sumed constant value of $7100 \mathrm{~K}$. Standard deviations accompany average values when more than one estimate is available.

We also obtained many du Pont echelle spectra of the well known very metal-poor $([\mathrm{Fe} / \mathrm{H}]<-2)$ subgiant HD 140283 which we used as a radial velocity standard. This star has been analyzed repeatedly with high spectral resolution, and the mean of entries in the PASTEL database (Soubiran et al. 2016) suggests $T_{\text {eff }}=5700 \mathrm{~K}, \log g=3.6$, and $[\mathrm{Fe} / \mathrm{H}]=-2.4$.

2 Spectra used in this investigation can be obtained at https://zenodo.org/record/2575102 
HD 140283 has much narrower absorption lines than RR Lyrae and other HB stars.

Observations of 24 very metal-poor RHB field stars stars were obtained with the Magellan MIKE spectrograph (Bernstein et al. 2003) in a mode that delivered $R \simeq 40,000$. The combination of Magellan's larger aperture and longer exposure times yielded $S / N>100$ at $5000 \AA$ in all cases. Reductions and analyses of the Magellan/MIKE spectra are discussed in Preston et al. (2006).

\subsection{Procedure}

We first derived estimates of line-of-sight velocity dispersion caused by the combination of macroturbulence and axial rotation, after removal of all other measurable line broadening sources. In this section we discuss how we avoid line damping problems and how we correct for instrumental broadening. Then in $\S 3$ we consider the effects of microturbulent and thermal broadening.

Cross correlation procedures developed to study galactic and stellar rotation (Simkin 1974, Tonry \& Davis 1979, 1981) are not useful for RR Lyrae stars. During typical RR Lyrae pulsation cycles temperatures vary by as much as $1000 \mathrm{~K}$, effective gravities vary by more than an order-of-magnitude, and turbulent velocities vary by amounts greater than their axial rotations. No stars in the CMDs of old metal-poor populations can serve as suitable templates for variable stars with these characteristics. Accordingly we developed a variant of the method adopted by Hosford et al. (2009), described in $\S 2.2 .1$ below.

\subsubsection{Elimination of Line Damping Effects}

For each spectrum we measured $F W H M$ and $E W$ of metal lines chosen from a list of some 200 unblended lines (mostly due to Fe I) in the wavelength range $\lambda \lambda 4100-5300 \AA$ that we judged to be unblended by inspection of the solar line identifications of Moore et al. (1966). These

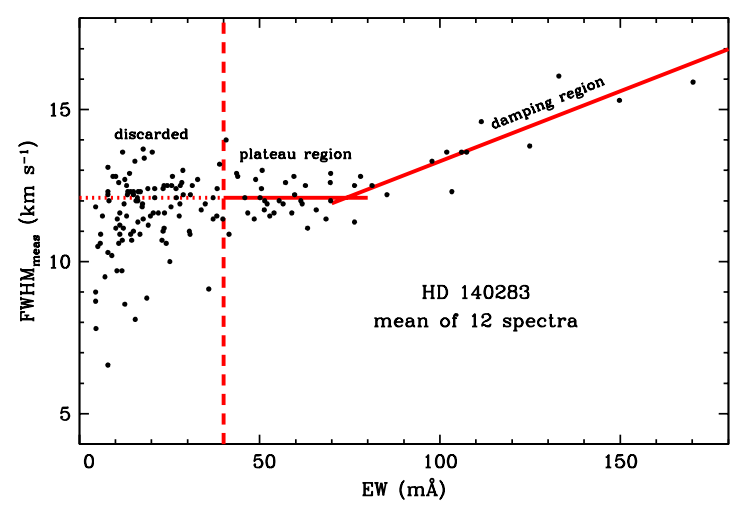

Figure 2. $F W H M_{o b s}$ versus $E W$ for lines measured in the du Pont echelle spectrum of HD 140283. These measurements were made on a spectrum formed by merger of 12 separate observations. There are three $E W$ regions: the discarded lines, $E W<40 \mathrm{~m} \AA$; the plateau lines, $40 \leq E W \leq 80 \mathrm{~m} \AA$; and damping lines, $E W>80 \mathrm{~m} \AA$. The division of $E W$ regions is discussed in the text.

lines are listed in Table 2. Less than half of them proved to be measurable in any particular spectrum. We expect that these lines are unblended in our spectra of RR Lyrae and RHB stars and in the spectrum of HD 140283. Measurements of these lines are labeled with subscript "meas". Then, using two methods described below, we culled from these measurements subsets of unsaturated "plateau" lines for each of the observed pulsation phases of each RR Lyrae star. The measurements of lines surviving this cut are labeled with subscript "plat".

(1) Our spectra with characteristic $S / N \sim 20$ to 30 and resolution of $R \sim 27,000$ are inadequate for direct detection of rotation profiles or analysis by Fourier methods. Hence, our recourse was to use a variant of the procedure of Hosford et al. (2009), who determined empirically the average minimum widths of weak metal lines as part of their study of lithium in metal-poor main sequence turnoff stars $\left(T_{\text {eff }} \sim 6200 \mathrm{~K}, \log g \sim 3.8\right)$. They demonstrated that in a plot of $E W$ versus $F W H M$, lines of their stars with $E W<90 \mathrm{~m} \AA$ lie on a "plateau" of constant $F W H M$ free of detectable damping wings; see their Figure 1. We 


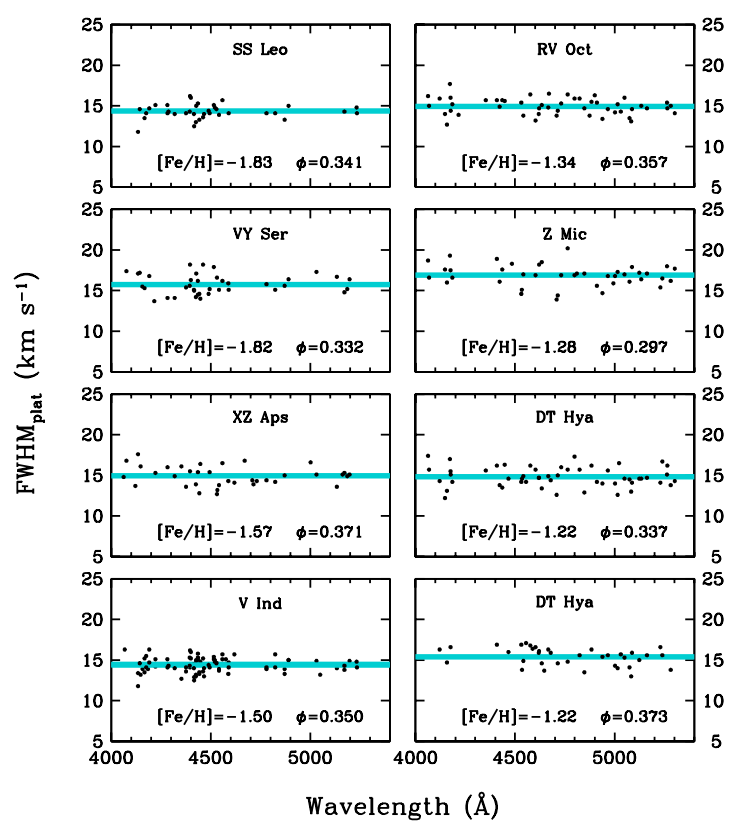

Figure 3. Plots of FWHM versus wavelength for plateau lines in metal-poor RRab stars, arranged in order of increasing $[\mathrm{Fe} / \mathrm{H}]$ from top-left to bottom-right.

found this procedure to be adequate for our purposes. We followed Hosford et al. (2009) in Figure 2 with a plot of $F W H M_{o b s}$ versus $E W$ for lines of Fe I and Fe II in HD 140283.

In somewhat hotter RR Lyrae stars, more metal poor by one to two orders-of-magnitude, depression of the continuum by hosts of weak unresolved lines is of greatly diminished importance. The effect of line opacity on the energy distributions of solar-type stars was treated in detail long ago by Melbourne (1960) and Wildey et al. (1962). Such stars display a marked decline in atomic line blanketing with increasing wavelength from $4000 \AA$ to 5500 AA. This is illustrated in Figure 1 of Wildey et al., which also shows the relative unimportance of line blanketing in the metal-poor $([\mathrm{Fe} / \mathrm{H}] \sim-2.0)$ subdwarf HD 19445.

All of our measures of $E W$ and $F W H M$ were made by use of the Gaussian profile fitting routine of IRAF/splot. In this procedure the computer operator chooses the local continuum level at each stellar line by interaction with a graphic display of the spec- trum. If unrecognized metallic lines were preferentially depressing the adopted continua at shorter wavelengths, we should see this effect as a positive correlation of FWHM with wavelength, but there are no such correlations in Figure 3, displayed for plateau lines at phases near $V_{\text {macrot }}$ minimum (phase $\phi \sim 0.38$ ) in the wavelength range 4000-5300 $\AA$. The stars in this montage are displayed from top-left to bottom-right in order of increasing $[\mathrm{Fe} / \mathrm{H}]$. It is instructive to note that the plateau value $F W H M_{\text {plat }} \sim 15 \mathrm{~km} \mathrm{~s}^{-1}$ is very nearly the same for all stars plotted in the montage except for $\mathrm{Z}$ Mic, a remarkable result that will be further documented in $\S 3.1 .1$ by the small dispersion of $V_{\text {macrot }}$ minima near phase 0.38 . We conclude that continuum placement is not a problem at phases near $V_{\text {macrot }}$ minimum, when the lines are narrowest and of greatest interest to us.

We used an upper bound to the plateau of $80 \mathrm{~m} \AA$ to identify the onset of increases of $F W H M_{\text {meas }}$ with increasing $E W$ due to damping in our RR Lyrae spectra. Because the Hosford et al. spectra had nearly double the resolving power $(R=47,000)$ and many times higher signal-to-noise $(\langle S / N\rangle \sim 100)$ than those of our RR Lyrae spectra, they were able to measure metal-lines as weak as $5 \mathrm{~m} \AA$. We did not trust our lines with $E W<40 \mathrm{~m} \AA$ to yield reliable $F W H M$ because of a "personal equation" effect that we found in our measurements of weak lines, i.e., those for which central depths are comparable to continuum noise of the spectra. Accidental negative noise in the wings of a line tends to produce broad absorption features that we frequently regarded as unmeasurable. However, accidental positive noise in the wings tends to produce spuriously narrow but easily measurable features. These effects become more important with decreasing spectral resolution and $S / N$, becoming particularly noticeable near minimum light (pulsation phase $\phi \sim 0.85$ ), when the lines become broad. Decisions about 

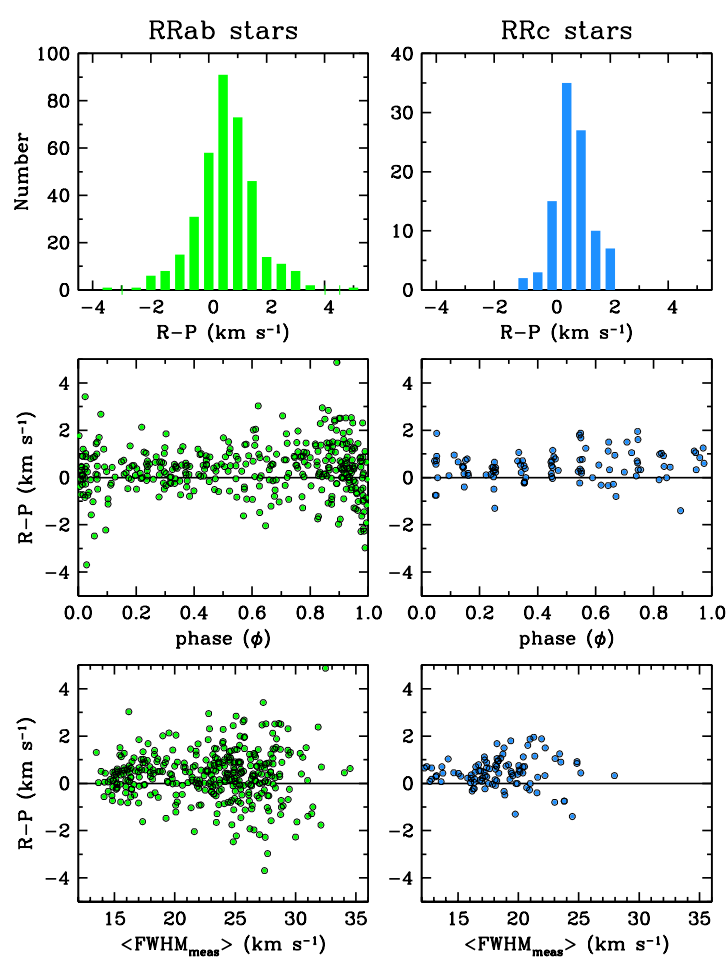

Figure 4. (top) Histogram of the difference $F W H M$ (damping regression at $E W=80 \mathrm{~m} \AA$ ) minus $F W H M$ (plateau) is strongly peaked near $0.5 \mathrm{~km} \mathrm{~s}^{-1}$ and (middle, bottom panels) varies little with pulsation phase and $F W H M$.

measurability will vary from one person to another, and for the same person at different times, hence "personal equation". The systematic effect arising from these biases, derived from measurements of hundreds of lines in eight RRab stars at all pulsation phases, is small but detectable $\left(1.07 \mathrm{~km} \mathrm{~s}^{-1} \pm 0.65 \mathrm{~km} \mathrm{~s}^{-1}\right)$. The effect varies with pulsation phase, going through a minimum near phase $\phi \simeq 0.35$ when the lines are intrinsically narrowest. To summarize, we calculated the average $F W H M_{\text {plat }}$ in the more restrictive domain $40 \mathrm{~m} \AA<E W<80 \mathrm{~m} \AA$.

(2) The damping regression for $E W>80 \mathrm{~m} \AA$ produces a second, independent estimate of $F W H M_{\text {plat }}$. This regression considers stronger, more accurately measured lines than those that lie on the plateau. Its numerical value, derived by the method of least squares for each spectrum, depends only slightly on the choice of the plateau $E W$ upper limit. The utility of this regression evaluated at $E W=80 \mathrm{~m} \AA$ as an estimator of the plateau value is simply an empirical fact, supported by the statistical comparisons of Regression and Plateau estimates in Figure 4. The top left panel of this figure is a histograms of 370 measured differences that we define as $F W H M(\mathrm{R}-\mathrm{P}) \equiv$ $F W H M$ (regression at $E W=80 \mathrm{~m} \AA$ ) minus $F W H M$ (plateau) for RRab stars binned at intervals of $0.5 \mathrm{~km} \mathrm{~s}^{-1}$. The average difference, $\langle F W H M(\mathrm{R}-\mathrm{P})\rangle=0.35 \pm 0.05 \mathrm{~km} \mathrm{~s}^{-1}$, is smaller than the measurement errors. The lower two panels of Figure 4 show that there is little variation of the difference with either pulsation phase or FWHM. Which of the two estimates is more reliable depends in a complicated way on $S / N$ and metallicity. The regression values are valuable for observations made during rising light and for metal-rich stars, situations in which we frequently are unable to measure weak lines.

We adopt the average of these two estimates as our final value of $F W H M_{\text {plat }}$ when both are available.

\subsubsection{Correction for Instrumental Line Broadening}

Instrumental broadening is comparable to the intrinsic widths of metal lines in our RR Lyrae spectra, so careful removal of it is important for the success of our analysis. We used the profiles of emission lines produced by a ThoriumArgon hollow cathode tube (hereafter ThAr) to remove the effects of instrumental broadening. The ThAr exposures were obtained by equipment and procedures described below.

The optical paths of starlight and ThAr light for the du Pont echelle are nearly identical except for central obscuration of some stellar light by the Cassegrain secondary mirror. A lens with the focal ratio of the telescope projects an image of the ThAr hollow cathode onto the entrance aperture of the spectrograph by a motor-operated mirror inserted into the tele- 
scope beam. ThAr spectra were obtained before and after observations at each position of the telescope. When we followed a star for lengthy intervals of time, we recorded the ThAr spectrum at intervals of 20 to 60 minutes; time intervals between ThAr exposures varied as required by various combinations of airmass, declination, and hour angle. We co-added successive pairs of ThAr exposures to make wavelength-calibrated extractions of stellar spectra obtained between successive pairs.

The temperature dependence of the focal adjustment of the echelle spectrograph is wellcalibrated. This calibration was employed to alter the focus of the spectrograph on those rare occasions when significant temperature variation during the night required its use.

Following the suggestion of an anonymous referee, we also measured the $F W H M$ of the [O I] $5577 \AA$ airglow line, which lies some $200 \AA$ longward of the upper limit of the spectral region used in our analyses, on all of the stellar spectra obtained on a dozen nights in the years 2006 through 2012. We compare these airglow measurements with measurements made of ThAr spectra obtained on these same nights in an Appendix.

We approximated corrections for all line broadening processes by use of the additive property, $\sigma_{a}=\left(\sigma_{b}^{2}+\sigma_{c}^{2}\right)^{\frac{1}{2}}$, of the Maxwell velocity distribution, in which $a, b, c$ are parameters of the Gaussian error function. We assume that for RR Lyrae stars the turbulent velocities on all length scales are isotropic throughout the metallic line-forming regions. To begin, we remove the effect of instrumental broadening, characterized by $F W H M_{\text {inst }}$, from average values of $F W H M_{\text {plat }}$ to obtain $F W H M_{\text {unsat }}$, according to

$F W H M_{\text {unsat }}=\left(\left\langle F W H M_{\text {plat }}\right\rangle^{2}-\left\langle F W H M_{\text {inst }}\right\rangle^{2}\right)^{\frac{1}{2}}$ in which $\left\langle F W H M_{\text {inst }}\right\rangle$ is an average value described below. We then convert $F W H M_{\text {unsat }}$ to a Maxwellian velocity dispersion by use of $\sigma_{\text {unsat }}=(2 \sqrt{\ln 2})^{-1} F W H M_{\text {unsat }}$. All further corrections are made in units of line-of sight velocity dispersion $\sigma$.

Removal of instrumental broadening was made to the average value $\left\langle F W H M_{\text {plat }}\right\rangle$ rather than to values of individual lines, because some measured widths of unsaturated lines may be narrower than $\left\langle F W H M_{\text {inst }}\right\rangle$. These narrowest lines belong in the average of a normal distribution, but they cannot be corrected for instrumental broadening by equation (1).

$F W H M_{\text {inst }}$ was evaluated as follows: We measured 591 FWHM values of some four dozen lines in each of 14 Thorium-Argon (ThAr) spectra chosen at random from spectra gathered in the years 2006-2012. Details of this investigation are given in the Appendix. We used the average value of all these measurements, $\left\langle F W H M_{\text {inst }}\right\rangle=11.36 \pm 0.52 \mathrm{~km} \mathrm{~s}^{-1}$ in equation (1) to calculate $F W H M_{\text {unsat }}$ for each RR Lyrae spectrum. This velocity corresponds to resolving power $R=26,700 \pm 200$, very close to the value of $R \simeq 27,000$ usually adopted for the du Pont echelle spectrograph.

\section{3. $V_{\text {macrot }}$ FROM MEASUREMENTS OF EW AND FWH $M_{\text {unsat }}$ FOR RRAB STARS}

For the calibration stars in Table 1 we have enough spectra to construct $F W H M_{\text {meas }}$ versus $E W$ relations throughout their pulsation cycles. For each star we combined observations made over several years into small phase intervals $(\sim 0.05 P)$ to increase $S / N$. The average numbers of phase bins for MP and MR stars are 22 and 18, respectively. For each spectrum we used IRAF $/$ splot $^{3}$ to measure $E W$ and $F W H M_{\text {meas }}$ for all usable lines in our list. Results from these

3 IRAF is distributed by the National Optical Astronomy Observatory, which is operated by the Association of Universities for Research in Astronomy (AURA) 


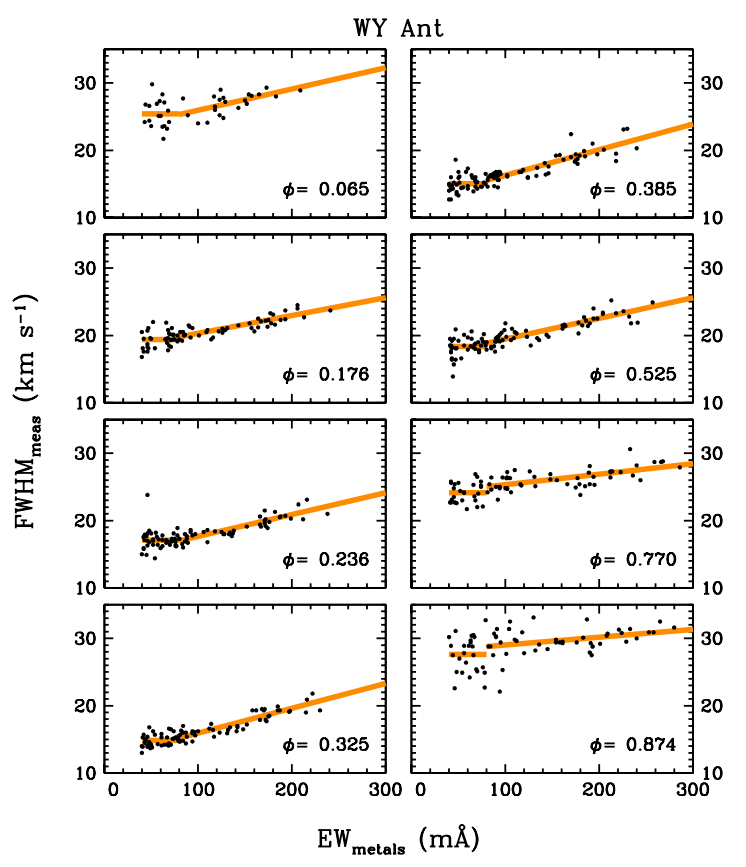

Figure 5. Montage showing how plateau $F W H M_{o b s}$ $(40 \leq E W \leq 80 \mathrm{~m} \AA)$ varies during the pulsation cycle of star WY Ant. Pulsation phases (relative to $\phi=0.0$ at maximum light) are shown in the bottom right corner of each panel.

measurements are listed columns 6 through 9 of Table 1.

From the $F W H M_{\text {meas }}$ versus $E W$ diagrams, direct evidence of the variation of macroturbulence with pulsation phase is easily seen. In Figure 5 we illustrate this variation for WY Ant with diagrams for eight phases during its pulsation cycle. The $F W H M_{\text {meas }}$ plateau value is high $\left(\sim 23 \mathrm{~km} \mathrm{~s}^{-1}\right)$ immediately after maximum light $(\phi=0.065)$; see the top left panel of Figure 5. It reaches a minimum $\left(\sim 15 \mathrm{~km} \mathrm{~s}^{-1}\right)$ in the bottom left panel, and increases steadily during the remainder of declining light shown in the right panels.

The $F W H M_{\text {plat }}$ subsets of these measurements were converted to $F W H M_{\text {unsat }}$ by equation (1), and from these to $V_{\text {macrot }}$. The $F W H M_{\text {plat }}$ value is similar to the damping

under cooperative agreement with the National Science Foundation. regression value at all phases, as shown in the middle panels of Figure 4. In Table 1 we provide estimates of the minimum value of $V_{\text {macrot }}$ for each star, calculated as the average $V_{\text {macrot }}$ for observations that lie in the phase interval $0.30<\phi<0.45$.

\subsection{The Behaviors of Macroturbulence and Microturbulence in RRab stars \\ 3.1.1. The Stable RRab stars}

Microturbulence, produced by motions on length scales smaller than the photon mean free path, broadens all spectral features but for weak lines (on the linear portion of the COG) the EWs are unchanged. Microturbulence will desaturate lines on the damping portion of the COG, thus increasing their $E W \mathrm{~s}$. Fortunately, $V_{m i c}$ can be derived from spectrum analysis (Gray 2008) with standard codes (e.g. $\mathrm{MOOG}^{4}$, Sneden 1973). We used the microturbulence values of For et al. (2011b) in our calculations. Macroturbulence, produced by motions on length scales large compared to the photon mean free path, acts to broaden all absorption lines. Macroturbulent velocities exceed microturbulent velocities of Richardson's (1920) eddies using the Siedentopf model of turbulent convection (Woolley \& Stibbs 1953, Ruediger 1989) to which we subscribe.

After computing $\sigma_{\text {unsat }}=(2 \sqrt{\ln 2})^{-1} F W H M_{\text {unsat }}$ for each spectrum of each star, we removed microturbulent and thermal velocity dispersions. These quantities were taken directly or computed from the atmospheric parameters for RRab stars derived by For et al. (2011b) and Chadid et al. (2017). In Figure 6 we show the variations in $T_{\text {eff }}, \log g, V_{m i c}$, and $V_{t h}$ as functions of pulsation phase for these stars. The gravities (panel b) are based on ionization equilibrium constraints only, as discussed in For et al. and Chadid et al.. Their values are shown

4 http://www.as.utexas.edu/ chris/moog.html 

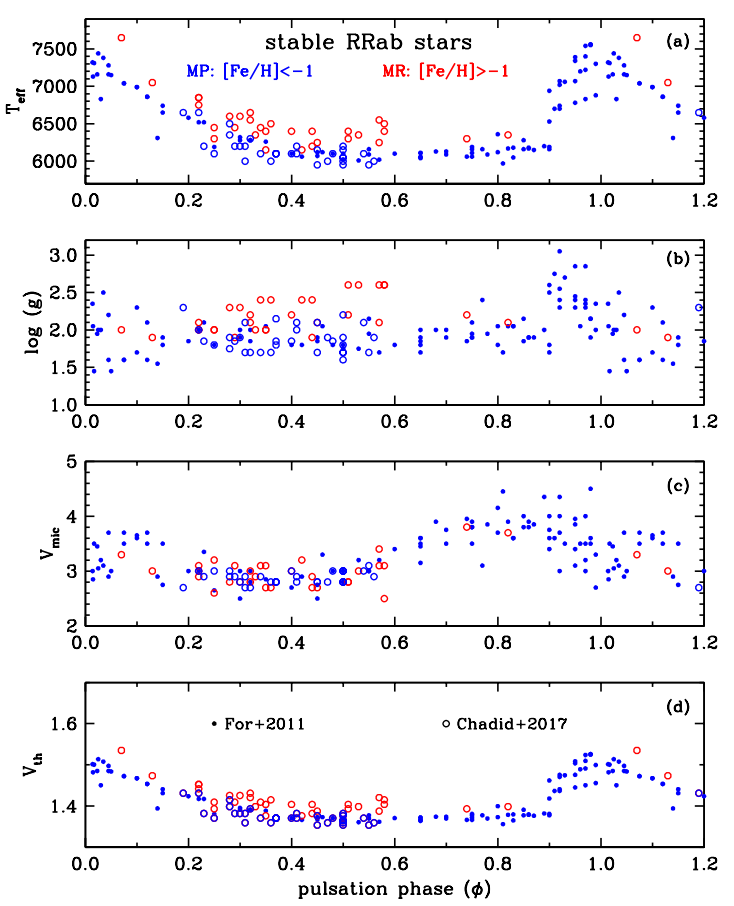

Figure 6. Variations with pulsation phase $\phi$ of $T_{\text {eff }}$ (panel a), $\log g$ (panel b), $V_{\text {mic }}$ (panel c), and $v_{t h}$ (panel d) for the RRab stars studied by For et al. (2011b) (dots) and Chadid et al. (2017) (open circles). Blue symbols denote MP stars and red symbols denote MR stars.

here only for completeness, as we will not use them in our subsequent calculations. Additionally, all quantities show significant scatter in the phase domains of rising and maximum light $(0.8<\phi<1.1)$; these parts of the pulsational cycles will not be used here.

We computed thermal velocities from $V_{t h}=(2 \mathrm{kT} / \mathrm{m})^{\frac{1}{2}}$, adopting $\mathrm{m}=54 \mathrm{amu}^{5}$, and these values are shown in panel (d) of Figure 6. These velocities are nearly constant, ranging only over $1.38 \lesssim V_{t h} \lesssim 1.53$, due to the modest RRab temperature variations (panel a) that are muted by the square root function. Over most of the pulsational cycles $V_{t h} \simeq 1.4 \mathrm{~km} \mathrm{~s}^{-1}$. Additionally, the microturbulent velocities shown in panel (c) are consistently larger: $V_{\text {mic }} \simeq 2 V_{t h}$ at all phases, so we have assumed a simgle micro-

$554 \mathrm{amu}$ is the mean value of the atomic masses of lines participating in the velocity calculations.
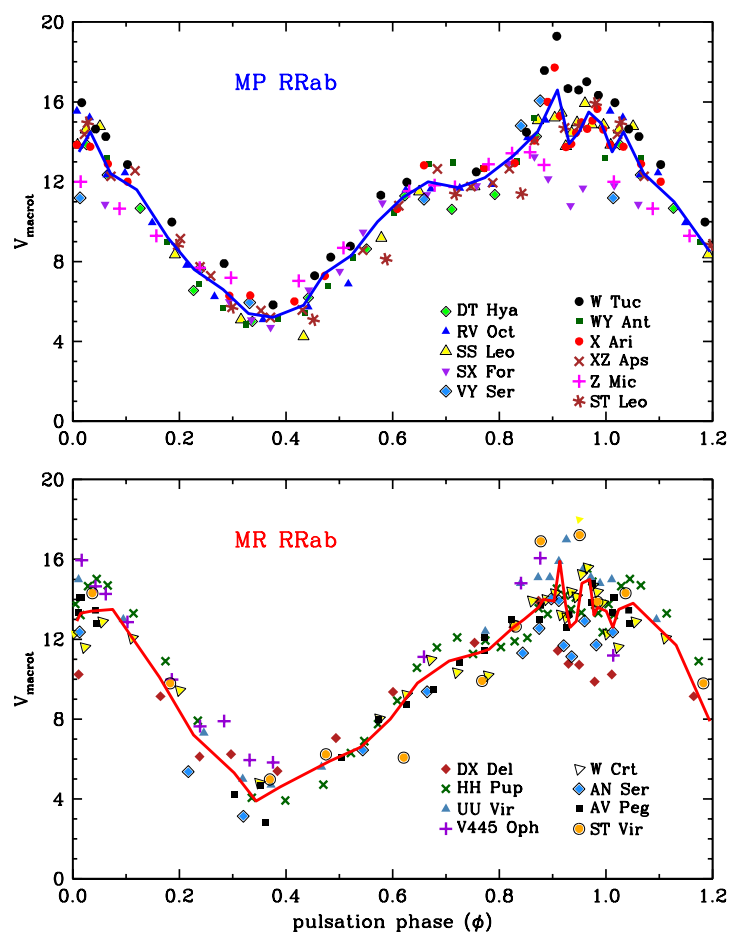

Figure 7. Variations of $V_{\text {macrot }}$ with phase for MP (top panel) and MR (bottom panel) RRab stars. Solid curves are mean variations as described in the text.

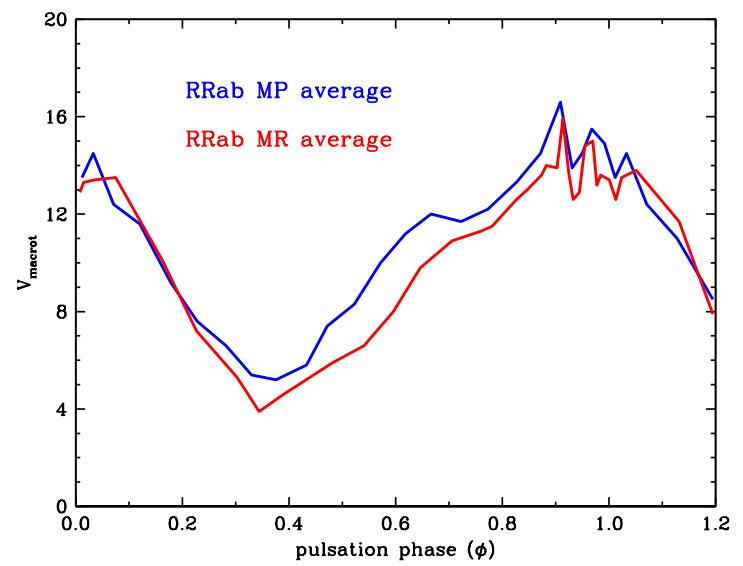

Figure 8. Average variations of $V_{\text {macrot }}$ with phase for MP (blue) and MR (red) RRab stars. The minimum value of $V_{\text {macrot }}$ / for MR stars is significantly lower than that for MP stars and the rising branch is shifted to later phase.

turbulent velocity of $2.8 \mathrm{~km} \mathrm{~s}^{-1}$ in all subsequent calculations. We use these data to plot $V_{\text {macrot }}=\left(\sigma_{\text {unsat }}^{2}-\sigma_{\text {mic }}^{2}-\sigma_{\text {th }}^{2}\right)^{\frac{1}{2}}$ versus phase in Figure 7 . The top and bottom panels contain, respectively, data for MP and MR stars. 
We also calculated average variations of $V_{\text {macrot }}$ with phase, the solid curves in Figure 7 , as follows. We sorted all the data by phase and then calculated averages $\langle\phi\rangle$ and $\left\langle V_{\text {macrot }}\right\rangle$ for data binned in successive groups of 10 measurements. We superpose the mean MP and MR curves without the individual data points in Figure 8. Minimum $V_{\text {macrot }}$ values for all the RR Lyrae stars in this investigation and for the MP RHB stars to be discussed in $\$ 5$ are presented in Table 1. The minimum averages, computed from data for each RRab in the phase interval $0.30<\phi<0.45$, are $5.31 \pm 0.13 \mathrm{~km} \mathrm{~s}^{-1}$ and $4.40 \pm 0.22 \mathrm{~km} \mathrm{~s}^{-1}$, respectively. These averages differ by an amount three times larger than the sum of their mean probable errors. Furthermore, the persistent offset of the two variations after phase $\phi=0.5$ in Figure 8 indicates a small but real difference between the behaviors of $V_{\text {macrot }}$ in MP and MR stars.

Although we can only claim that the $V_{\text {macrot }}$ minima in Figure 7 provide upper limits on $V_{\text {rot }} \sin (i)$ for the MP and MR RRab, the small scatter of individual stars near these minima strongly suggests that macroturbulence, not rotation, dominates the minimum values. Random inclination of rotation axes alone should create dispersion in the minimum values, and we are uncomfortable with the notion that all RRab stars rotate at the same speed: thus, the actual rotations of RRab stars may lie well below our calculated upper limits.

\subsubsection{The Blazhko RRab stars}

Complex variations of metallic radial velocities and line widths of Blazhko RRab stars near their light maxima (RV minima) defy summary description. One example, UV Oct, is shown in Figure 9. The incomplete sampling of Blazhko phases for UV Oct is typical for the Blazhko stars in our data set. Because phases near maximum light contribute nothing to our exploration of axial rotation, we restricted our attention to observations made during declining light
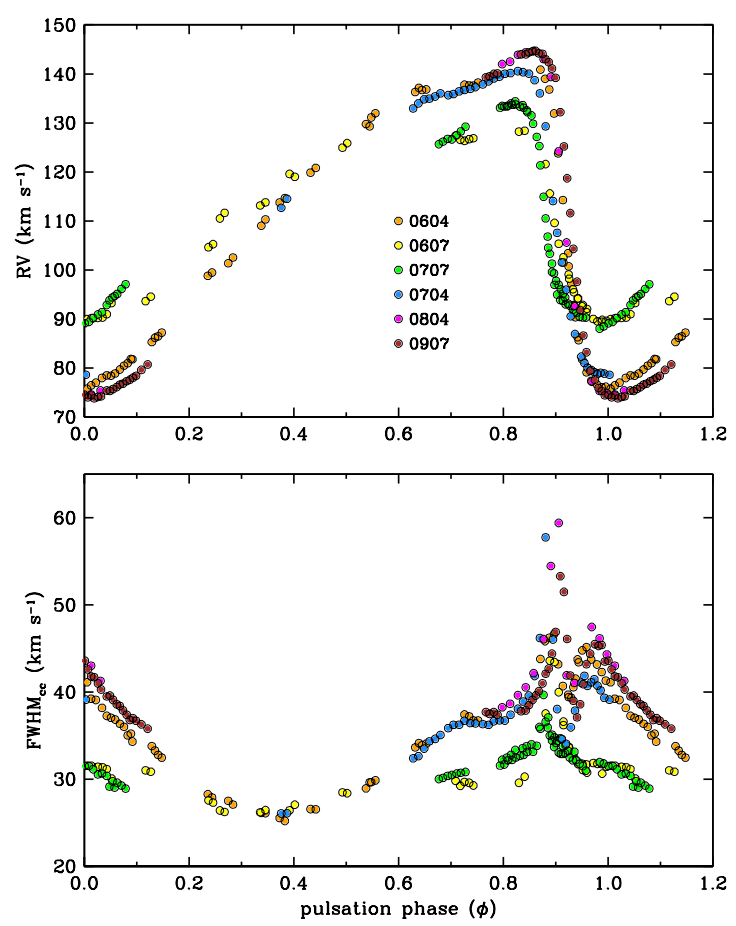

Figure 9. Radial velocity RV (top panel) and $F W H M_{c c}$ of the cross correlation function (bottom panel) versus phase for the RRab Blazhko star UV Oct. Data were obtained by cross correlation with a template spectrum of CS 22874-009 (Preston \& Sneden 2000). The colors indicate different observing seasons, denoted in the figure legend as yymm, where yy are the last digits of the year and $\mathrm{mm}$ are the digits of the month, e.g., 0604 is April of 2006.

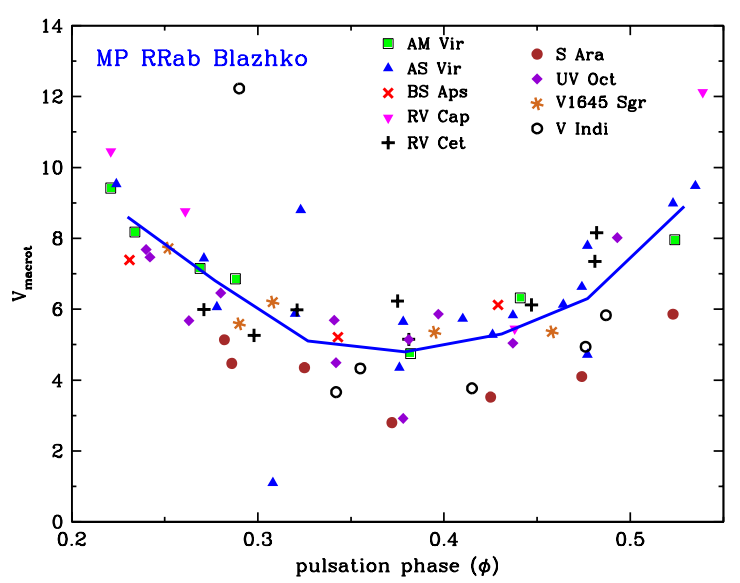

Figure 10. $V_{\text {macrot }}$ versus phase for nine Blazhko RRab stars. The solid black curve denotes mean points calculated for data in successive 0.05 phase intervals. 


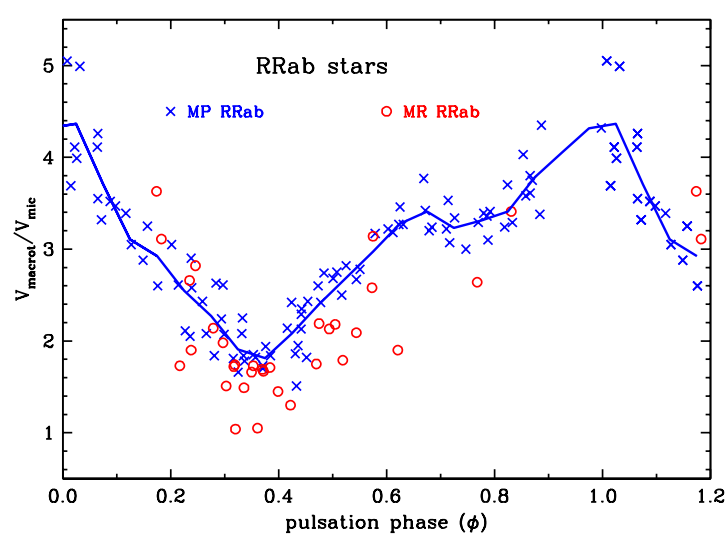

Figure 11. Variation with phase of $V_{\text {macrot }} / V_{\text {mic }}$, an empirical spectrum of turbulence, for the MP and MR RRab stars. The blue curve represents the mean trend for the MP RRab. Its values were computed in phase intervals of $\Delta \phi=0.05$; see Table 4 .

$(0.20<\phi<0.55)$, where line-widths of stable RRab stars achieve their minimum values. The ephemerides in Table 3 produce phases of radial velocity minima near zero for our Blazhko variables (Chadid \& Preston 2013, Preston, unpublished). Values of $V_{\text {macrot }}$ plotted versus these phases are displayed in Figure 10. Modest phase corrections, adopted by eye-inspection and listed in column 4 of Table 3, were added to phases calculated with these ephemerides to reduce horizontal scatter in this figure. Black line segments connect mean points of observations calculated at intervals of $0.05 \mathrm{P}$. The lowest mean point, $V_{\text {macrot }}=4.77 \pm 0.32 \mathrm{~km} \mathrm{~s}^{-1}$, occurring at $\phi=0.38$, is slightly smaller than the mean value for stable RRab stars, $\left\langle V_{\text {macrot }}\right\rangle=5.31 \pm 0.13 \mathrm{~km} \mathrm{~s}^{-1}$. Stable RRab and Blazhko RRab stars yield the same upper limit on axial rotation of $5 \pm 1 \mathrm{~km} \mathrm{~s}^{-1}$.

\subsection{An Empirical "Spectrum of Turbulence" in RRab Stars}

For stable RRab stars we combine the variations of macroturbulence (Figure 7) and microturbulence (Figure 6) by forming their ratio. The microturbulent velocities for these stars were derived by For et al. (2011b) and Chadid et al. (2017) from phase-combined spectra and so there are fewer of these values than those of $V_{\text {macrot }}$. This is especially true of the Chadid et al. results, which were usually based on only 3 or 4 combined phase points. We matched phases of $V_{\text {macrot }}$ and $V_{\text {mic }}$ data for as many points per star as possible, interpolating the $V_{m i c}$ values where possible and not attempting any extrapolations into phase intervals not covered by both data sets. The results of this exercise are displayed in Figure 11, where MP and MR stars are distinguished by different symbols and colors. Solid line segments connect mean values of data points calculated in phase intervals of $0.05 \mathrm{P}$. These mean points are listed in Table 4 . This variation of the $V_{\text {macrot }} / V_{\text {mic }}$ ratio with phase is in accord with the minimal requirements of Richardson (1920), Kolmogorov (1941), and the Siedentopf model (Woolley \& Stibbs 1953) at all phases, namely, that $V_{\text {mac }}$ always exceeds $V_{\text {mic }}$. We offer this ratio as a primitive empirical description of a "spectrum of turbulence", defined by the two length scales associated with macro- and micro-turbulence, in RRab atmospheres during their pulsation cycles.

\section{4. $V_{\text {macrot }}$ FROM EWS AND FWHMS OF RRC STARS}

The measurements of EW and $F W H M_{\text {meas }}$ for the RRc stars and the conversion of these measurements to estimates of $V_{\text {macrot }}$ proceeded in the same manner followed for RRab stars in $\S 3$. We chose lines for measurement from the same line list. Because the RRc stars are systematically hotter than the RRab stars, metallic absorption lines are systematically weaker. However, the agreement between plateau and regression estimates shown in Figure 4 is hardly impaired: $\langle F W H M(\mathrm{R}-\mathrm{P})\rangle=0.46 \pm 0.06 \mathrm{~km} \mathrm{~s}^{-1}$ for RRc stars, is only $0.11 \mathrm{~km} \mathrm{~s}^{-1}$ larger than the value derived for RRab stars, $0.35 \pm 0.05 \mathrm{~km} \mathrm{~s}^{-1}$. 

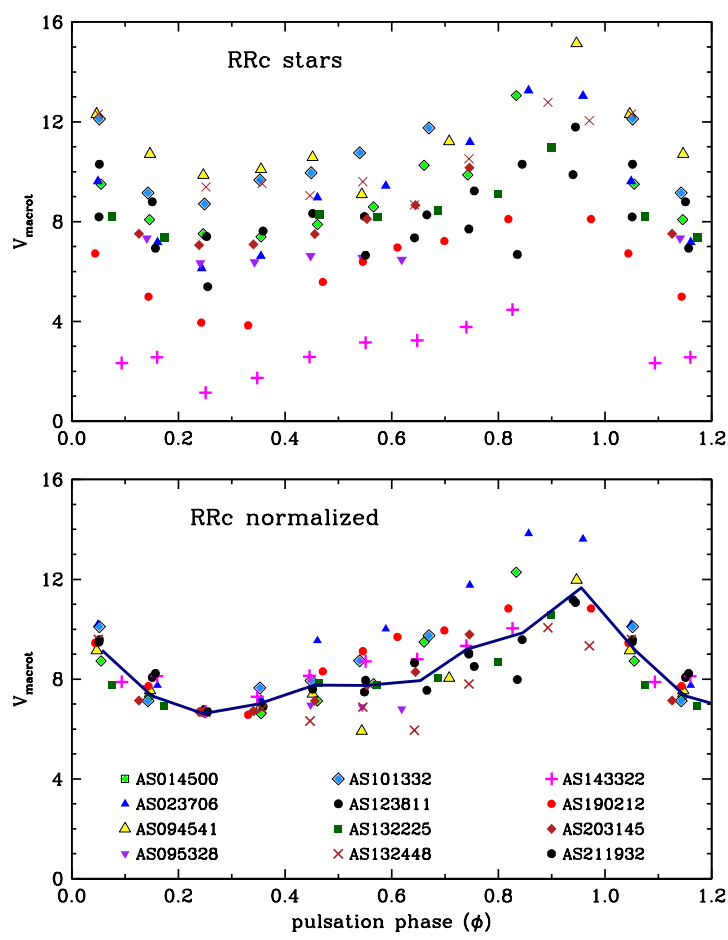

Figure 12. Top panel: $V_{\text {macrot }}$ versus phase for 12 RRc stars. Bottom panel: the same data as in the top panel, but with the $V_{\text {macrot }}$ values in each star arbitrarily shifted to coincide with the data point for AS1433 at phase 0.25 . The solid curve connects data points phaseaveraged in groups of five.

The variations of $V_{\text {macrot }}$ with phase for RRc stars, presented in the top panel of Figure 12, differ markedly from the uniform behavior of the RRab stars in Figure 7. Minimum values of $V_{\text {macrot }}$ occur at earlier phases, near phase $\phi=0.3$, and range from 2 to $12 \mathrm{~km} \mathrm{~s}^{-1}$. Maximum values occur near maximum light. We create a characteristic variation of $V_{\text {macrot }}$ with phase in the bottom panel of Figure 12 by use of additive constants that superpose data for all stars near phase $\phi \simeq 0.25$ at $V_{\text {macrot }}=6.62 \mathrm{~km} \mathrm{~s}^{-1}$, the mean value for the sample. This exercise is an instructive, albeit mathematically incorrect, way to combine the data. The amplitude of the solid black curve that connects mean points of individual observations is $\sim 4 \mathrm{~km} \mathrm{~s}^{-1}$, much smaller than the amplitude for RRab stars $\left(\sim 10 \mathrm{~km} \mathrm{~s}^{-1}\right)$.
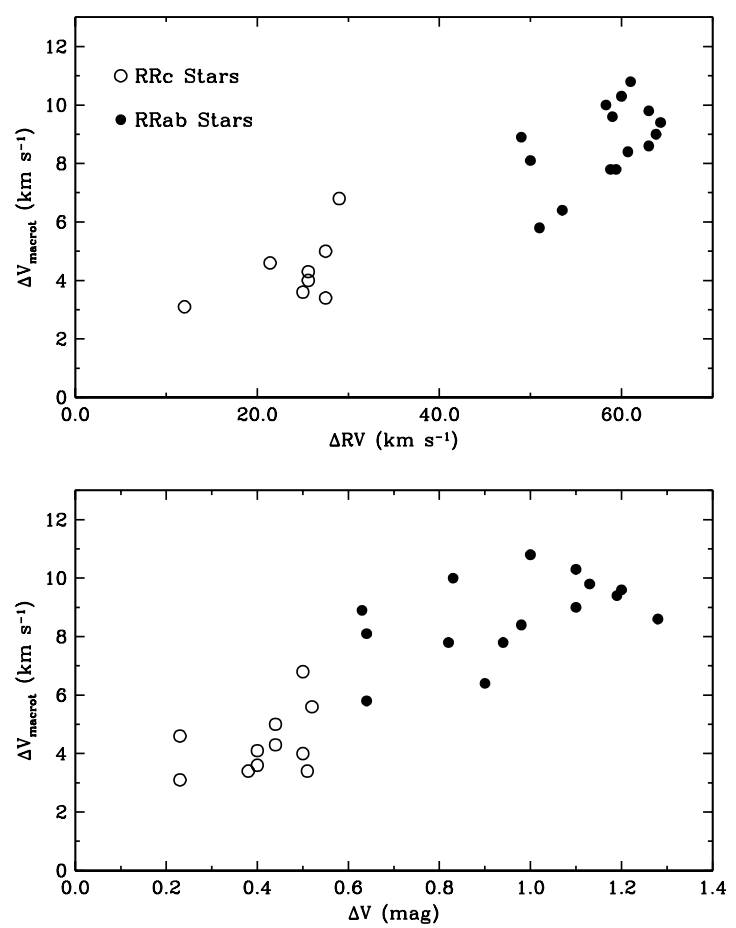

Figure 13. Correlations of $\Delta V_{\text {macrot }}$ amplitude (the difference beteween maximum and minimum $V_{\text {macrot }}$ values) with (top panel) RV amplitude and (bottom panel) visual light amplitude during pulsation cycles.

We can understand the behavior of the RRc stars in the top panel of Figure 12 by noting that macroturbulent velocity amplitude, the difference between maximum and minimum values of $V_{\text {macrot }}$ achieved in a pulsation cycle, scales with pulsation amplitude for all RR Lyraes as shown by the regressions in Figure 13. Accordingly, we imagine that less macroturbulence in RRc stars with their smaller pulsation amplitudes permits detection of smaller axial rotations in RRc stars than those encountered among the RRab. AS190212-4639 (4 $\left.\mathrm{km} \mathrm{s}^{-1}\right)$ and AS143322-0418 ( 1 $\left.\mathrm{km} \mathrm{s}^{-1}\right)$ are outstanding examples. The large upper limit of $V_{\text {macrot }}$ $\left(\sim 12 \mathrm{~km} \mathrm{~s}^{-1}\right)$ for RRc suggests that BHB rapid rotators adjacent to the instability strip lose their surface angular momentum during evolution through the RRc portion of the instability strip, a possibliity worthy of further exploration with better data. 


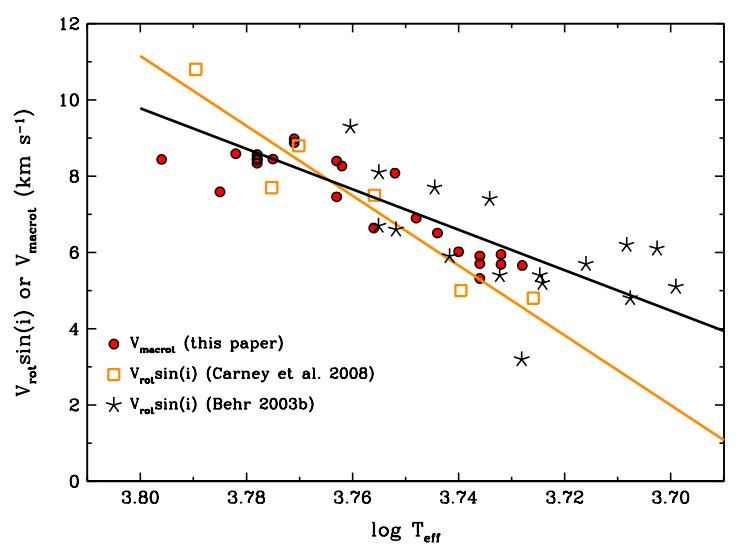

Figure 14. $V_{\text {macrot }}$ values for RHB stars of this study and the $V \sin i$ values of Behr (2003b) and Carney et al. (2008) are plotted versus $\log T_{\text {eff }}$.

\section{5. $V_{\text {macrot }}$ FROM EWS AND FWHMS OF METAL-POOR RHB STARS}

We used Magellan MIKE echelle spectra (outlined in $\S 2$ ) to estimate $V_{\text {macrot }}$ for twenty-two $\mathrm{MP}$ red horizontal branch stars. We adopted $F W H M_{\text {inst }}=7.20 \mathrm{~km} \mathrm{~s}^{-1}$ derived from ThAr lines to remove instrumental broadening, and we borrowed $V_{t h}$ and $V_{m i c}$ data from Preston et al. (2006) to calculate values of $V_{\text {macrot }}$.

These $V_{\text {macrot }}$ values are systematically 1 to 2 $\mathrm{km} \mathrm{s}^{-1}$ larger than those of the RRab stars and they decline smoothly with decreasing temperature along the RHB portion of the horizontal branch. This decline is shared by the values of $V_{\text {rot }} \sin (i)$ derived by Behr (2003b) and Carney et al. (2008) as illustrated in Figure 14. Behr (2003b) removed macro-turbulent broadening from his measured line widths by the conservative assumption that macro-turbulent velocities equal the micro-turbulent velocities derived from his spectrum analyses. The mean value and standard deviation of these values for the Behr sample presented here are $2.11 \pm 0.44 \mathrm{~km} \mathrm{~s}^{-1}$. Carney et al. (2008) derived much larger macro-turbulent velocities (6 to $12 \mathrm{~km} \mathrm{~s}^{-1}$ ) from Fourier transform analyses of line profiles provided by high resolution, high $\mathrm{S} / \mathrm{N}$ spectra of six RHB stars. Carney et al. remark that "rotation and macro-turbulence play

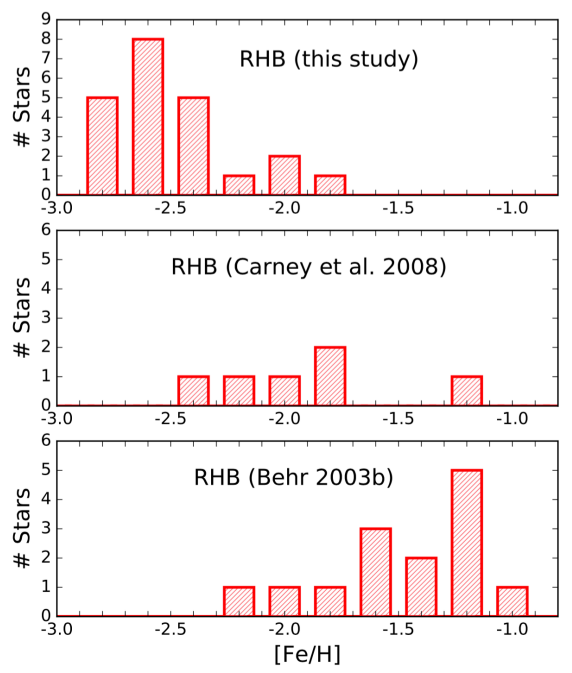

Figure 15. Histograms of the $[\mathrm{Fe} / \mathrm{H}]$ distributions of three metal-poor RHB samples. Strong selection effects are evident.

comparable roles in the line broadening of the observed RHB stars", whereas Behr, by virtue of his assumed small macro-turbulent velocity, found that rotation dominates in his sample. The data assembled in Figure 3 of Grassitelli et al. (2015) support the conclusions of Carney et al. Comparison of the only two stars, HD 25532 and HD 184266, common to the Behr and Carney et al. samples, are presented in the Table 5. We suspect that the larger $V_{\text {rot }} \sin (i)$ values of Behr in this table arise, at least in part, from his assumed low macro-turbulent velocities. Because our $V_{\text {macrot }}$ values lie comfortably between/along the regression lines of Carney et al. and Behr in Figure 14 we conclude that our $V_{\text {macrot }}$ values are very nearly $V_{\text {rot }} \sin (i)$ values, i.e., the macro-turbulent velocities of our RHB stars lie below our limit of detectability.

We omitted HD $195636\left(V_{\text {rot }} \sin (i)=20.6 \mathrm{~km} \mathrm{~s}^{-1}\right.$, $T_{\text {eff }}=5399 \mathrm{~K},[\mathrm{Fe} / \mathrm{H}]=-2.74$; Behr 2003b) from Figure 14 because it lies so far from the locus of all other metal-poor RHB. The star is an indubitable metal-poor, giant, rapid rotator (see discussion by Preston 1997).

We seek an explanation for the small values of macro-turbulent velocities in our RHB sample in the work of Tanner et al. (2013) and 
Grassitelli et al. (2016), who report that at a given effective temperature the optically thin super-adiabatic layers of model atmospheres with higher metallicity possess larger convective velocities. This is an abundance effect: higher atmospheric opacities produce optically thin layers in regions of lower gas density. Their calculations induced us to construct the metallicity distributions of the three RHB samples, which we display as histograms in Figure 15. It is evident at a glance that major selection effects are present. The RHB stars of the present study were discovered in the course of high resolution spectroscopic investigations (Preston et al. 2006; Roederer et al. 2014) of the most metal-poor stars, $[\mathrm{Fe} / \mathrm{H}] \leq-2$, identified in the survey of Beers et al. (1992), while the samples of Behr (2003b) and Carney et al. (2008) were chosen by exploration of more abundanceinclusive, photometrically-defined samples. The content of the histograms is summarized in Table 6 .

The mean $[\mathrm{Fe} / \mathrm{H}]$ values of this study and that of Behr (2003b) differ by one full orderof-magnitude. The sample fractions with $[\mathrm{Fe} / \mathrm{H}]>-1.7$ in these three studies range from 0.0 to 0.8 . This comparison of our very metalpoor sample with that of Carney et al. (2008) supports the conclusion of Tanner et al. (2013): macro-turbulent velocities in the optically thin layers that produce metal absorption lines increase with increasing metallicity. Perhaps this presentation will stimulate renewed interest in abundance-dependent macro-turbulence on the horizontal branch.

\section{DISCUSSION}

In this paper we have combined high resolution spectra of RR Lyrae and RHB stars collected at Las Campanas Observatory over three decades with literature studies of RHB and $\mathrm{BHB}$ stars to derive rotation rates or meaningful upper limits for stars along the horizontal

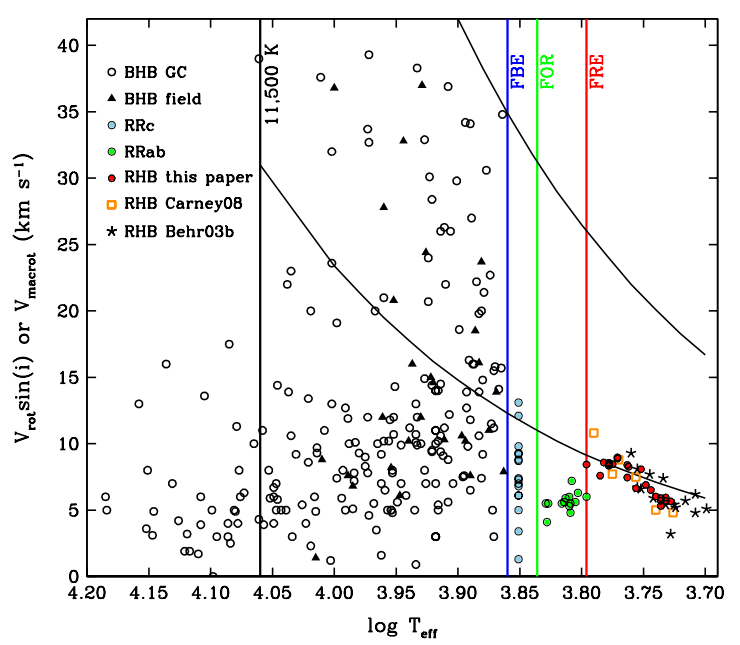

Figure 16. Variation of $V_{r o t} \sin (i)$ (BHB stars) and $V_{\text {macrot }}\left(\mathrm{RR}\right.$ Lyrae and RHB stars) with $\log \left(T_{\text {eff }}\right)$ along the metal-poor horizontal branch. The black vertical line denotes the Grundahl limit at 11,500 K. For the instability strip the vertical lines are: the fundamental blue edge (FBE, blue); the first overtone red edge (FOR, green), and the fundamental red edge (FRE, red). Black parabolic trajectories define the regions of the instability strip traversed by BHB rapid rotators that evolve with constant Luminosity and constant angular momentum $\left(V_{\text {rot }} \propto T_{\mathrm{eff}}^{2}\right)$.

branch. In Figure 16 we present a summary plot, showing $V_{\text {macrot }}$ or $V_{\text {rot }} \sin (i)$ as a function of effective temperature along the HB. The astonishing variations of measured rotational velocity with $T_{\text {eff }}$ clearly indicate that surface angular momentum cannot be a proper measure of total angular momentum of stars along the HB.

To begin discussion of Figure 16, we recall that the large values of $V_{\text {rot }} \sin (i)$ for many metal-poor BHB stars first encountered by Peterson et al. (1995) at $T_{\text {eff }}<11500 \mathrm{~K}$ were totally unexpected in view of the small projected rotational velocities $\left(V_{\text {rot }} \sin (i)<5 \mathrm{~km} \mathrm{~s}^{-1}\right)$ of their evolutionary antecedents in globular clusters (Lucatello \& Gratton 2003). Then, the abrupt decline in rotation at the blue edge of the instability strip (Peterson et al. 1996) was an additional surprise, appropriately labeled a "conundrum" by Preston (2011) and Preston 

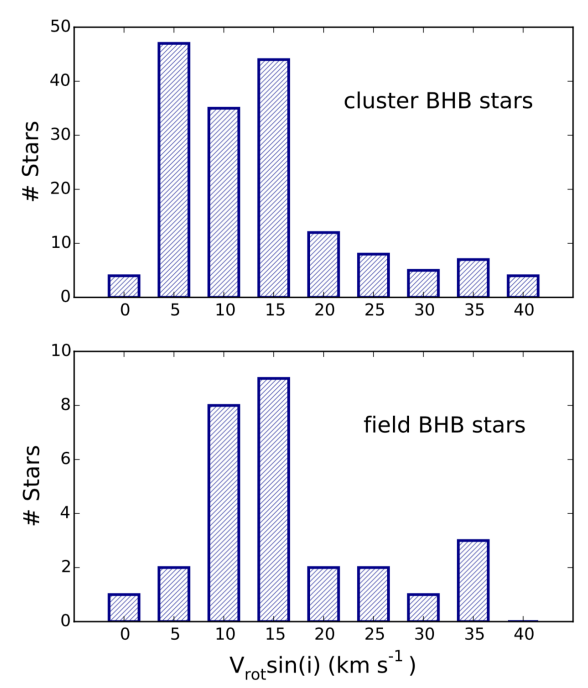

Figure 17. The $V_{\text {rot }} \sin (i)$ distributions of globular cluster (top panel) and field (bottom panel) BHB stars in the compilation of Cortés et al. (2009).

\& Chadid (2013). This investigation reduces the upper limit of RRab rotation from 10 to $5 \mathrm{~km} \mathrm{~s}^{-1}$, and additionally suggests that rapid $\mathrm{BHB}$ rotation disappears during RRc evolution. Should the arguments of Tanner et al. (2013) apply to RR Lyrae, the upper limit on RR Lyrae rotation would be further reduced.

Extant $V_{\text {rot }} \sin (i)$ distributions differ from cluster to cluster in the Galactic halo. However, whether field and globular cluster HB star samples are drawn from the same parent population is an open question. The $V_{\text {rot }} \sin (i)$ distributions of the Cortés et al. (2009) compilations of field and cluster HB stars are similar, as noted previously by Cortés et al. and shown in Figure 17. This similarity encouraged us to include the cluster HB stars in Figure 16. Only a handful of the $\sim 150$ known Galactic globular clusters have been investigated for rotation, and the field sample is limited to HB stars near the solar circle, so it is premature to draw firm conclusions about this issue.

The use of "during" in the description of RRc evolution deserves comment because of the complicated nature of $\mathrm{HB}$ evolution in and near the instability strip. This is best appreciated

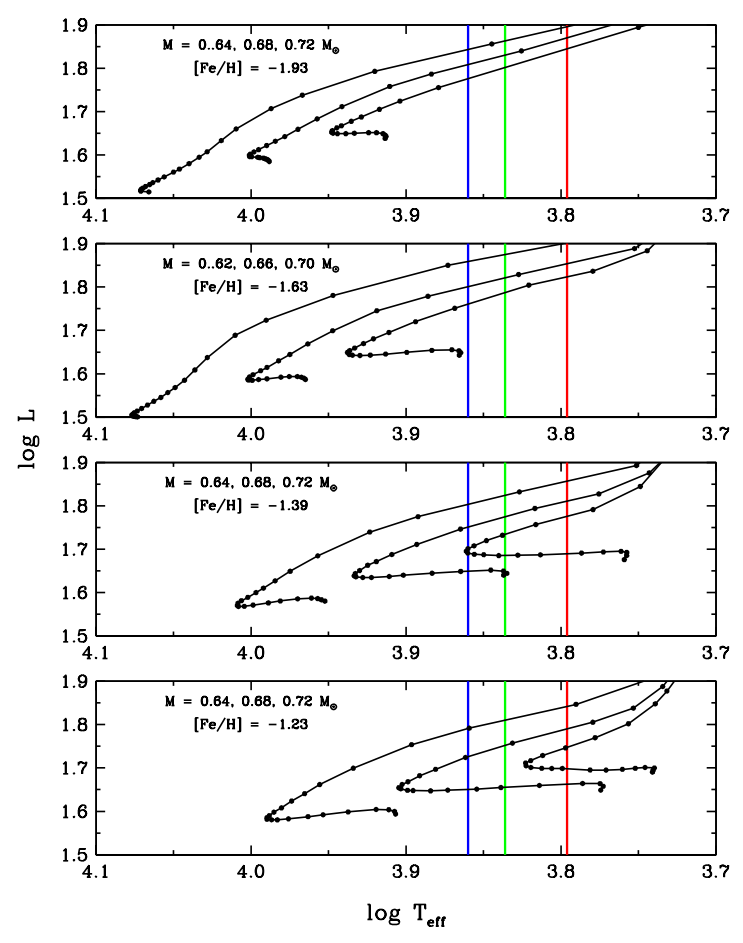

Figure 18. Evolutionary tracks for HB stars with masses and abundances that bracket the models adopted by VandenBerg et al. (2016) for the globular clusters M5, M15, and M92. Blue, green, and red vertical lines mark the fundamental blue edge, first overtone red edge, and fundamental red edge of the instability strip.

by inspection of the evolutionary tracks of Lee \& Demarque (1990) in Figure 18 plotted for $\mathrm{Y}=0.23$ with masses and abundances that bracket the models adopted for the metal-poor globular clusters M3, M15, and M92 by VandenBerg et al. (2016). For nearly all of the models plotted in Figure 18, approximately horizontal evolution at near-constant Luminosity toward higher effective temperatures occupies $\sim 2 / 3$ of their horizontal branch lifetimes (see Figure 1 of de Santis \& Cassisi (1999)).

Second parameter issues play no role in our study. Evolution from the Zero Age Horizontal Branch (ZAHB) to higher temperature, the "blue loops" in Figure 18 is the result of a hydrogen-burning phenomenon common to all HB evolutionary sequences (Sweigart 1987, Lee \& Demarque 1990, Castellani et al. 1991, Dorman 1992, Dotter et al. 2007) for reasons dis- 

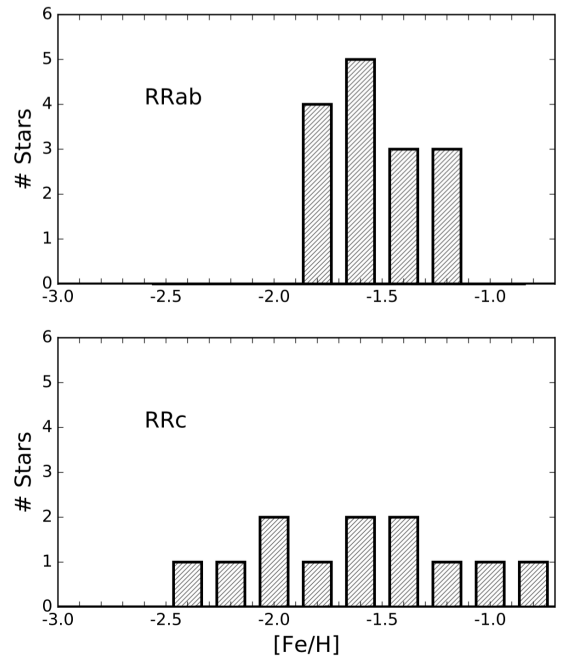

Figure 19. Abundance distributions of the RR Lyrae stars studied in this paper.

cussed in some detail by Sweigart, by Dorman, and by Dotter et al.. Early on Rood (1973) realized that these loops could not provide the range in $\mathrm{HB}$ color distributions required of a second parameter. However, the blue loops would act to blur the pattern of $V_{\text {macrot }}$ values of RHB and RR Lyrae stars seen in Figure 16, were ZAHB stars to retain their initial surface angular momenta during migration from the RHB and/or RR Lyrae domains toward the BHB. For $[\mathrm{Fe} / \mathrm{H}]<-1.6$ evolution proceeds steadily from the BHB through the instability strip to the $\mathrm{RHB}$ and on to the AGB. However, for stars with $[\mathrm{Fe} / \mathrm{H}]>-1.6$, evolution can be quite complicated. As a consequence of stochastic mass loss, an RRc star-to-be may arrive on the ZAHB variously as an RHB, RRab, RRc or BHB star, all of these evolving to higher temperatures for most of their HB lifetimes. From the metallicity distributions of our RR Lyrae samples shown in Figure 19 we see that roughly half of them, those with $[\mathrm{Fe} / \mathrm{H}]<-1.6$, evolve monotonically from the BHB through the instability strip to the AGB. At higher metallicity a star can arrive on the ZAHB as an RHB star, evolve through the RRab and RRc states to the BHB and then go back across the instability strip to the AGB.
However, Figure 19 indicates that the stars retain no memory of their initial ZAHB locations.

We observe a decline in mean $V_{\text {rot }} \sin (i)$ during the transition from $\mathrm{BHB}$ to RRc; then a gentle rise in the transition from RRab to RHB, followed by a decline during subsequent RHB evolution toward the AGB. Absent a mechanism for altering the total angular momentum of $\mathrm{HB}$ stars, we attribute these regularities in behavior of $V_{\text {rot }} \sin (i)$ among the RR Lyrae and RHB stars as indications that angular momentum is continuously being redistributed within these stars on time scales short, $\sim 10^{7} \mathrm{y}$, compared to HB lifetimes. Sills \& Pinsonneault (2000) discuss schemes for such redistribution.

As regards removal of surface angular momentum that might accompany mass loss during RR Lyrae evolution, the only credible investigation of which we are aware is the 3D numerical simulation of Stellingwerf (2013), which produced a hot corona with outflow. Stellingwerf did not estimate the mass loss rate, and we are loathe to speculate in this matter. Whether or not these notions are correct, we believe that they are legitimate food for thought worthy of presentation to theoreticians.

Finally, we note the inclusion in Figure 16 of HD 195636 with $\log T_{\text {eff }}=3.732, V_{\text {rot }} \sin (i)=20.6 \mathrm{~km} \mathrm{~s}^{-1}$, $[\mathrm{Fe} / \mathrm{H}]=-2.7$ (Behr 2003b) lying far above all other RHB stars. We deliberately excluded this star from the discussion of $\S 5$ simply because it is such an egregious outrider. A long history of investigations, summarized by Preston (1997), confirm that it is a very metal-poor RHB or AGB star with lines abnormally broad for its presumed evolutionary state. Further speculation about the history of HD 195636 is beyond the scope of this paper.

We thank all the Las Campanas Observatory support personnel for their help during the course of our endeavor, with particular regards to several du Pont telescope operators 


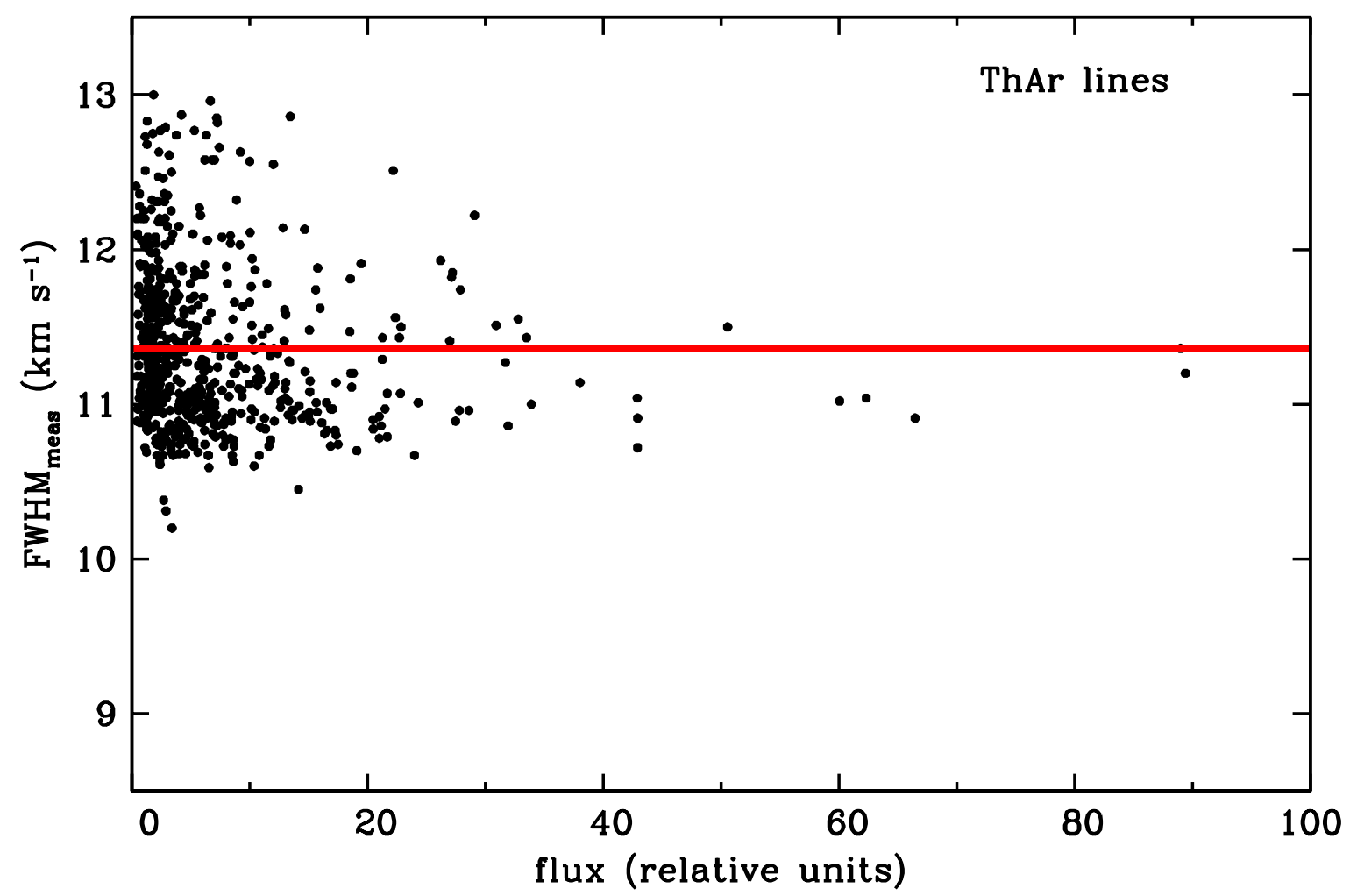

Figure 20. FWHM of ThAr emission lines plotted versus the relative integrated line fluxes.

for their efforts in assisting with the observations required to produce this paper. We also thank Michel Breger, Marcio Catelan, Bill Cochran, and Phillip MacQueen for helpful comments that improved our presentation. This work has been supported in part by NSF grant AST1616040 to C.S. and by the University of Texas Rex G. Baker, Jr. Centennial Research Endowment.

\section{APPENDIX}

\section{A. ESTIMATES OF $F W H M_{\text {inst }}$ OF THE DUPONT ECHELLE SPECTROGRAPH}

We explore two ways to estimate the instrumental width, $F W H M_{\text {inst }}$, of the duPont echelle spectrograph.

(1) Using procedures described in $\S 2.2 .2$, we made 591 measurements of $E W$ (arbitrary flux units) and $F W H M\left(\mathrm{~km} \mathrm{~s}^{-1}\right)$ in six echelle orders of fourteen ThAr spectra chosen at random from observations made during observing runs in the years 2006, 2007, 2009, 2011, and 2012. The results are plotted in Figure 20 as a plot of $F W H M$ versus $E W$, where the horizontal line represents the average value, $11.36 \pm 0.52 \mathrm{~km} \mathrm{~s}^{-1}$, of all the measurements, The average and standard deviation for the 151 strongest lines are reduced only slightly to $11.25 \pm 0.45 \mathrm{~km} \mathrm{~s}^{-1}$. Inspection of Figure 20 reveals an obvious skew toward larger $F W H M$ values. This asymmetry arises from degradation of the echelle instrumental profile with increasing angular distance from the optical axis of the spectrograph. This degradation is due to optical aberrations and to inevitable slight tilt of the CCD chip with respect to the focal surface of the spectrograph camera.

We tested the hypothesis that skew is introduced by position-dependent aberrations by using the $(\mathrm{x}, \mathrm{y})$ pixel coordinates of ThAr the lines in the CCD image frames to calculate the radial distance 


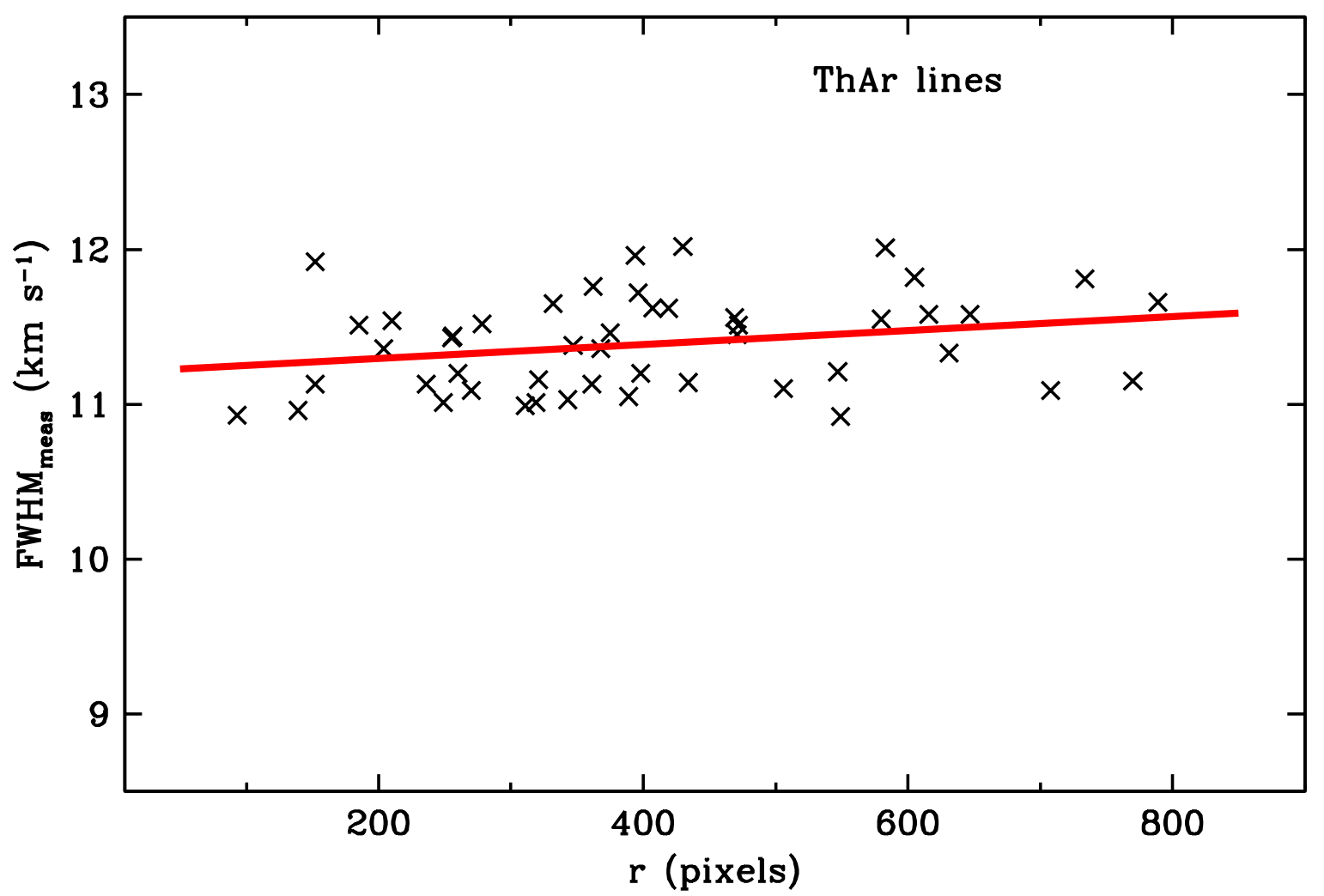

Figure 21. FWHM of ThAr lines plotted versus radial pixel distance from the center of the CCD chip. The red line is a linear regression derived from the plotted data points.

$\mathrm{r}(\mathrm{x}, \mathrm{y})$ of each line from the central coordinates of the CCD chip $(\mathrm{x}=1024, \mathrm{y}=1024)$. The results of this exercise are shown in Figure 21. We found that $\langle F W H M\rangle$ increases with $\mathrm{r}(\mathrm{x}, \mathrm{y})$ by $\sim 0.4 \mathrm{~km} \mathrm{~s}^{-1}$ over the usable range of $\mathrm{r}(\mathrm{x}, \mathrm{y})$, in qualitative agreement with expectations.

(2) Following the suggestion of an anonymous referee, we measured $F W H M$ of the airglow line [O I] $5577 \AA$ in 792 spectra of RR Lyrae stars observed in the years 2006-2012. This single telluric line is not ideal for our purposes, because it lies at a single location on the CCD chip some $1000 \AA$ (20 echelle orders) longward of the bulk of our ThAr and stellar line measurements. Our measurements are displayed in Figure 22 as a plot of $F W H M$ versus $E W$ of the airglow emission in units of normalized stellar continua. The [ $\left[\begin{array}{ll}\mathrm{O}\end{array}\right]$ emission feature is characteristically weak in our spectra because of our short $(<600 \mathrm{~s})$ exposure times. The red horizontal line in this figure represents the average of all measurements, $10.86 \pm 0.62 \mathrm{~km} \mathrm{~s}^{-1}$. The scatter of individual $F W H M$ about this mean value is free of skew because it is located at the same near-central location on the CCD chip in all spectra. The mean FWHM of the [O I] distribution is $0.50 \mathrm{~km} \mathrm{~s}^{-1}$ smaller than that of the ThAr lines. No reasonable assumptions about thermal broadening of [O I] $5577 \AA$ in the Earths atmosphere or Th lines in hollow cathode lamps can produce this difference, the cause of which remains unknown to us.

We consider how our conclusions about $V_{\text {macrot }}$ depend on our choice of $F W H M_{\text {inst }}$ by calculating the difference in $V_{\text {macrot }}(5577)-V_{\text {macrot }}(\mathrm{ThAr})$ as a function of phase. In Figure 23 we display these differences for the twelve RRab stars identified in the figure legend. In this exercise we used $10.86 \mathrm{~km} \mathrm{~s}^{-1}$, the average for all measurements of [O I] $5577 \AA$, and $11.36 \mathrm{~km} \mathrm{~s}^{-1}$, the average for 


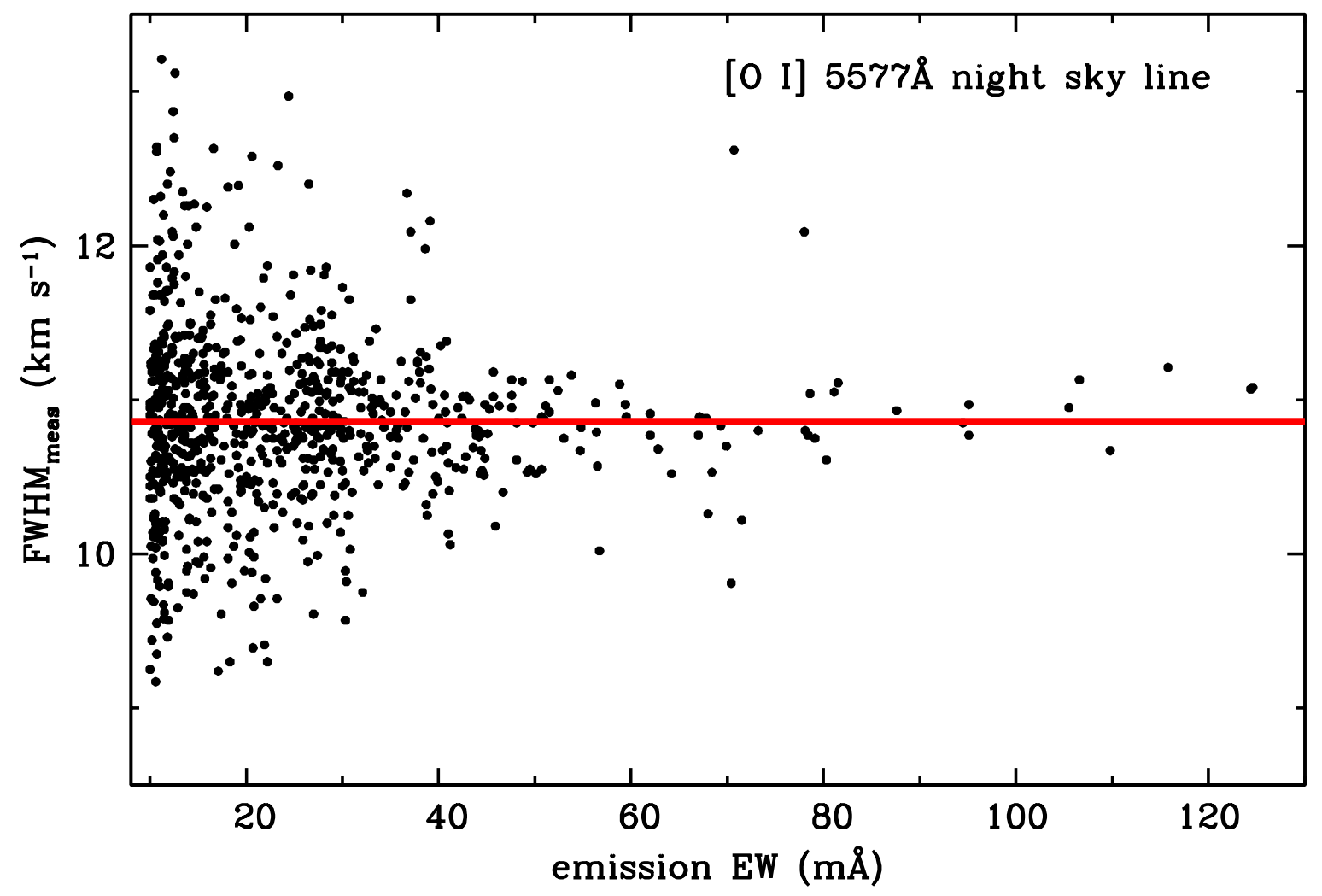

Figure 22. $F W H M_{\text {meas }}$ of the night sky [O I] 5577 emission line plotted versus its $E W$ in units of normalized stellar fluxes.

all measurements of ThAr lines. The smaller $F W H M_{\text {inst }}$ derived from [O I] $5577 \AA$ produces larger $V_{\text {macrot }}$ values. These differences in $V_{\text {macrot }}$, which vary with pulsation phase are generally smaller than $0.4 \mathrm{kmsec}$ near phase $\phi=0.38$, the phase we use to set the upper limit on $V_{\text {rot }} \sin (i)$. This difference, $0.4 \mathrm{~km} \mathrm{~s}^{-1}$, provides an independent estimate of uncertainty in our upper bound of $5 \mathrm{~km} \mathrm{~s}^{-1}$ for the $V_{r o t} \sin (i)$ of metal-poor RRab stars. We prefer the larger $F W H M$ derived from the ThAr lines, because these lines are distributed more nearly like those of the metallic absorption lines which occur at random locations in all spectral orders.

(3) Finally, consider the narrow-lined HD 140283, our radial velocity standard. It has a measured $F W H M_{\text {meas }}=12.15 \mathrm{~km} \mathrm{~s}^{-1}$ that narrowly exceeds our adopted $F W H M_{\text {inst }}=11.36 \mathrm{~km} \mathrm{~s}^{-1}$. We calculate $V_{\text {macrot }}=1.6 \mathrm{~km} \mathrm{~s}^{-1}$ for HD 140283 by use of these FWHM values, $T_{\text {eff }}=5750 \mathrm{~K}$, and $V_{\text {mic }}=1.50 \mathrm{~km} \mathrm{~s}^{-1}$. Collet et al. (2009) used their 3D hydrodynamic code to derive $V_{\text {rot }} \sin (i)=2.58$ $\mathrm{km} \mathrm{s}^{-1}$ for HD140283. The difference between their value and ours, $1.0 \mathrm{~km} \mathrm{~s}^{-1}$, is consistent with our estimate of the uncertainty in our calculations, and it is unclear which of these two values is to be preferred.

\section{REFERENCES}

Beers, T. C., Preston, G. W., \& Shectman, S. A. 1992, AJ, 103, 1987

Behr, B. B. 2003a, ApJS, 149, 67

-. 2003b, ApJS, 149, 101
Bernstein, R., Shectman, S. A., Gunnels, S. M., Mochnacki, S., \& Athey, A. E. 2003, in Society of Photo-Optical Instrumentation Engineers (SPIE) Conference Series, Vol. 4841, Instrument Design and Performance for Optical/Infrared Ground-based Telescopes, ed. M. Iye \& A. F. M. Moorwood, 1694-1704 


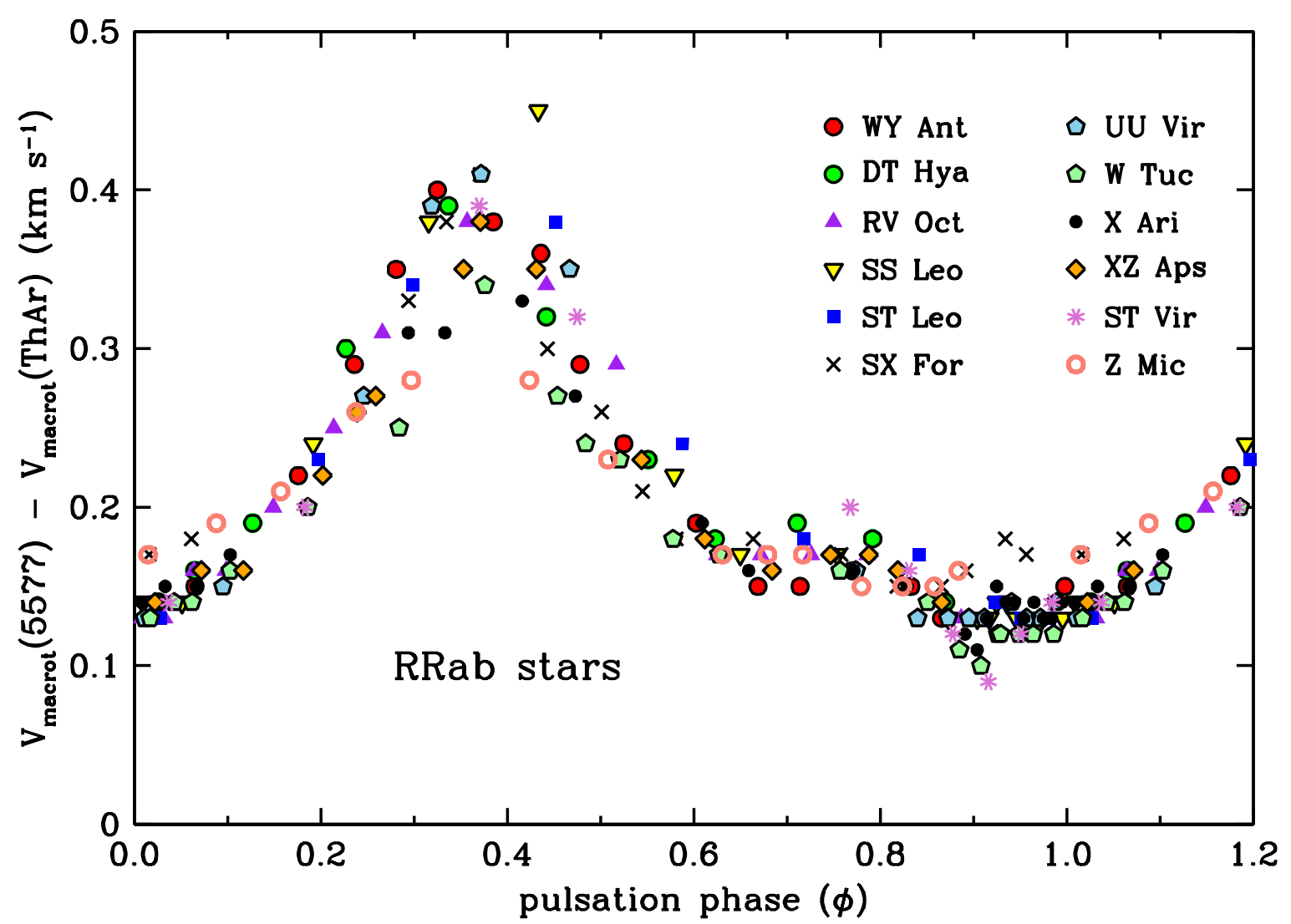

Figure 23. The increases in $V_{\text {macrot }}$, if $F W H M_{\text {inst }}$ is reduced from $11.36 \mathrm{~km} \mathrm{~s}^{-1}$ to $10.86 \mathrm{~km} \mathrm{~s}^{-1}$, are plotted as a function of pulsation phase for 12 RRab stars.

Bersier, D., \& Burki, G. 1996, A\&A, 306, 417

Breitfellner, M. G., \& Gillet, D. 1993, A\&A, 277, 553

Carney, B. W., Gray, D. F., Yong, D., et al. 2008, AJ, 135, 892

Castellani, V., Chieffi, A., \& Pulone, L. 1991, ApJS, 76, 911

Chadid, M., \& Gillet, D. 1996, A\&A, 315, 475

Chadid, M., \& Preston, G. W. 2013, MNRAS, 434,552

Chadid, M., Sneden, C., \& Preston, G. W. 2017, ApJ, 835, 187

Collet, R., Asplund, M., \& Nissen, P. E. 2009,

Publications of the Astronomical Society of Australia, 26, 330

Cortés, C., Silva, J. R. P., Recio-Blanco, A., et al. 2009, ApJ, 704, 750

de Santis, R., \& Cassisi, S. 1999, MNRAS, 308, 97

Dorman, B. 1992, ApJS, 81, 221

Dotter, A., Chaboyer, B., Jevremović, D., et al. 2007, AJ, 134, 376

Fokin, A. B., Gillet, D., \& Chadid, M. 1999, A\&A, 344, 930
For, B.-Q., Preston, G. W., \& Sneden, C. 2011a, ApJS, 194, 38

For, B.-Q., Sneden, C., \& Preston, G. W. 2011b, ApJS, 197, 29

Grassitelli, L., Fossati, L., Langer, N., et al. 2015, A\&A, 584, L2

—. 2016, A\&A, 593, A14

Gray, D. F. 2008, The Observation and Analysis of Stellar Photospheres (Cambridge, UK:

Cambridge University Press)

Grundahl, F., Catelan, M., Landsman, W. B., Stetson, P. B., \& Andersen, M. I. 1999, ApJ, 524,242

Hosford, A., Ryan, S. G., García Pérez, A. E., Norris, J. E., \& Olive, K. A. 2009, A\&A, 493, 601

Kinman, T., Castelli, F., Cacciari, C., et al. 2000, A\&A, 364, 102

Kolmogorov, A. 1941, Akademiia Nauk SSSR Doklady, 30, 301

Lee, Y.-W., \& Demarque, P. 1990, ApJS, 73, 709

Lucatello, S., \& Gratton, R. G. 2003, A\&A, 406, 691 
Melbourne, W. G. 1960, ApJ, 132, 101

Michaud, G., Tarasick, D., Charland, Y., \& Pelletier, C. 1983, ApJ, 269, 239

Moore, C. E., Minnaert, M. G. J., \& Houtgast, J. 1966, The solar spectrum 2935 A to 8770 A (National Bureau of Standards Monograph, Washington: US Government Printing Office (USGPO))

Peterson, R. C., Carney, B. W., \& Latham, D. W. 1996, ApJL, 465, L47

Peterson, R. C., Rood, R. T., \& Crocker, D. A. 1995, ApJ, 453, 214

Preston, G. W. 1997, AJ, 113, 1860

—. 2011, AJ, 141, 6

Preston, G. W., \& Chadid, M. 2013, in EAS Publications Series, Vol. 63, EAS Publications Series, ed. G. Alecian, Y. Lebreton, O. Richard, \& G. Vauclair, 35-45

Preston, G. W., \& Sneden, C. 2000, AJ, 120, 1014

Preston, G. W., Sneden, C., Thompson, I. B., Shectman, S. A., \& Burley, G. S. 2006, AJ, 132, 85

Quievy, D., Charbonneau, P., Michaud, G., \& Richer, J. 2009, A\&A, 500, 1163

Recio-Blanco, A., Piotto, G., Aparicio, A., \& Renzini, A. 2004, A\&A, 417, 597

Richardson, L. F. 1920, Proceedings of the Royal Society of London Series A, 97, 354

Roederer, I. U., Preston, G. W., Thompson, I. B., et al. 2014, AJ, 147, 136

Rood, R. T. 1973, ApJ, 184, 815

Ruediger, G. 1989, Differential rotation and stellar convection. Sun and the solar stars (Berlin: Akademie Verlag, 1989)
Sills, A., \& Pinsonneault, M. H. 2000, ApJ, 540, 489

Simkin, S. M. 1974, A\&A, 31, 129

Skarka, M. 2014, MNRAS, 445, 1584

Sneden, C. 1973, ApJ, 184, 839

Sneden, C., Preston, G. W., Chadid, M., \& Adamów, M. 2017, ApJ, 848, 68

Sneden, C., Preston, G. W., Kollmeier, J. A., et al. 2018, AJ, 155, 45

Soubiran, C., Le Campion, J.-F., Brouillet, N., \& Chemin, L. 2016, A\&A, 591, A118

Stellingwerf, R. F. 2013, ArXiv e-prints, arXiv: 1310.0535

Stift, M. J., \& Gillet, D. 1994, in Astronomical Society of the Pacific Conference Series, Vol. 64, Cool Stars, Stellar Systems, and the Sun, ed. J.-P. Caillault, 735

Sweigart, A. V. 1987, ApJS, 65, 95

Szczygieł, D. M., \& Fabrycky, D. C. 2007, MNRAS, 377, 1263

Tanner, J. D., Basu, S., \& Demarque, P. 2013, ApJ, 778, 117

Théado, S., Alecian, G., LeBlanc, F., \& Vauclair, S. 2012, A\&A, 546, A100

Tonry, J., \& Davis, M. 1979, AJ, 84, 1511

Tonry, J. L., \& Davis, M. 1981, ApJ, 246, 666

VandenBerg, D. A., Denissenkov, P. A., \& Catelan, M. 2016, ApJ, 827, 2

Wildey, R. L., Burbidge, E. M., Sandage, A. R., \& Burbidge, G. R. 1962, ApJ, 135, 94

Woolley, R., \& Stibbs, D. W. N. 1953, The Outer Layers of a Star (Oxford: Clarendon Press) 
Table 1. The Stellar Sample

\begin{tabular}{|c|c|c|c|c|c|c|c|c|c|}
\hline Star Name & {$[\mathrm{Fe} / \mathrm{H}]$} & $\begin{array}{c}P \\
\text { days }\end{array}$ & $\begin{array}{l}H J D_{0} \\
\text { days }\end{array}$ & $\begin{array}{c}V \\
\max \text { light }\end{array}$ & $\begin{array}{c}\log \left(T_{\text {eff }}\right)^{\mathrm{a}} \\
(\mathrm{K})\end{array}$ & $\begin{array}{c}\left\langle F W H M_{\text {unsat }}\right\rangle \\
\mathrm{km} \mathrm{s}^{-1}\end{array}$ & $\begin{array}{c}\sigma\left(F W H M_{\text {unsat }}\right) \\
\mathrm{km} \mathrm{s}^{-1}\end{array}$ & $\begin{array}{c}\left\langle V_{\text {macrot }}\right\rangle \\
\mathrm{km} \mathrm{s}^{-1}\end{array}$ & $\begin{array}{c}\sigma\left(V_{\text {macrot }}\right) \\
\mathrm{km} \mathrm{s}^{-1}\end{array}$ \\
\hline \multicolumn{10}{|c|}{ MP RRab Calibration } \\
\hline WY Ant & -1.66 & 0.574344 & 3835.592 & 10.4 & 3.810 & 10.2 & 0.3 & 5.3 & 0.2 \\
\hline XZ Aps & -1.57 & 0.587266 & 3836.276 & 11.9 & 3.809 & 10.5 & 0.2 & 5.5 & 0.2 \\
\hline SW Aqr & -1.24 & 0.459303 & 1876.138 & 10.6 & 3.829 & 10.5 & $\cdots$ & 5.5 & $\cdots$ \\
\hline SX For & -1.62 & 0.605342 & 1870.406 & 10.9 & 3.805 & 10.7 & 1.2 & 5.6 & 0.8 \\
\hline VX Her & -1.60 & 0.455359 & 2699.996 & 10.1 & 3.828 & 8.6 & 0.6 & 4.1 & 0.5 \\
\hline DT Hya & -1.22 & 0.567978 & 3835.608 & 12.5 & 3.814 & 10.7 & 0.9 & 5.6 & 0.6 \\
\hline SS Leo & -1.83 & 0.626335 & 1873.056 & 10.5 & 3.809 & 9.5 & 0.6 & 4.8 & 0.4 \\
\hline AT Ser & -2.05 & 0.746568 & 5326.814 & 11.0 & $\cdots$ & 12.1 & 1.5 & 6.0 & 1.0 \\
\hline W Tuc & -1.64 & 0.642243 & 5454.570 & 10.9 & 3.810 & 11.2 & $\cdots$ & 6.0 & $\cdots$ \\
\hline \multicolumn{10}{|c|}{ MP RRab Blazhko ${ }^{c}$} \\
\hline BS Aps & -1.51 & 0.582560 & 3836.378 & 11.9 & 3.808 & 11.0 & 0.7 & 5.8 & 0.4 \\
\hline DN Aqr & -1.77 & 0.633766 & 5364.300 & 10.8 & 3.785 & 11.5 & 1.6 & 6.1 & 1.1 \\
\hline S Arab & $\cdots$ & 0.451883 & 2137.338 & 10.3 & 3.827 & 8.2 & 0.8 & 3.7 & 0.6 \\
\hline RV Cap & -1.72 & 0.447748 & 5328.400 & 10.6 & 3.825 & 10.6 & $\cdots$ & 5.6 & $\cdots$ \\
\hline RV Cet & -1.32 & 0.623421 & 5455.460 & 10.6 & 3.804 & 11.2 & 0.6 & 6.0 & 0.4 \\
\hline UV Oct & -1.69 & 0.542573 & 3836.795 & 9.2 & 3.815 & 9.9 & 1.3 & 5.0 & 0.9 \\
\hline V Ind & -1.62 & 0.479602 & 1873.466 & 9.6 & 3.825 & 8.6 & 0.4 & 4.1 & 0.3 \\
\hline DX Del & -0.52 & 0.472619 & 5323.417 & 9.8 & 3.822 & 11.2 & 0.6 & 5.9 & 0.4 \\
\hline V445 Oph & -0.05 & 0.397024 & 1939.030 & 10.5 & 3.831 & 9.3 & 0.8 & 4.6 & 0.6 \\
\hline AV Peg & -0.14 & 0.390382 & 5360.677 & 10.0 & 3.836 & 8.8 & 1.0 & 4.1 & 0.7 \\
\hline HH Pup & -0.95 & 0.390745 & 1869.661 & 10.6 & 3.837 & 8.5 & 0.5 & 4.0 & 0.1 \\
\hline AN Ser & -0.06 & 0.522072 & 2701.153 & 10.5 & 3.811 & 7.6 & $\cdots$ & 3.3 & $\cdots$ \\
\hline ST Vir & -0.85 & 0.410806 & 5323.225 & 11.0 & 3.806 & 10.0 & $\cdots$ & 5.1 & $\cdots$ \\
\hline UU Vir & -0.93 & 0.475609 & 1886.488 & 10.1 & 3.826 & 9.8 & 0.2 & 5.0 & 0.1 \\
\hline \multicolumn{10}{|c|}{ MP RRc Stable } \\
\hline as014500-3003 & -2.28 & 0.377380 & 1869.252 & 11.1 & 3.851 & 13.1 & 0.10 & 7.4 & 0.06 \\
\hline as023706-4257 & -1.88 & 0.311326 & 1869.352 & 8.8 & 3.851 & 11.4 & 0.38 & 6.1 & 0.26 \\
\hline as094541-0644 & -2.10 & 0.350225 & 1869.729 & 10.6 & 3.851 & 17.1 & 0.19 & 9.8 & 0.12 \\
\hline as095328+0203 & -1.76 & 0.324697 & 1869.701 & 9.9 & 3.851 & 11.4 & 0.03 & 6.1 & 0.02 \\
\hline as101332-0702 & -1.73 & 0.316000 & 1869.668 & 10.8 & 3.851 & 15.8 & 0.77 & 9.2 & 0.48 \\
\hline
\end{tabular}

Table 1 continued 
Table 1 (continued)

\begin{tabular}{|c|c|c|c|c|c|c|c|c|c|}
\hline Star Name & {$[\mathrm{Fe} / \mathrm{H}]$} & $\begin{array}{c}P \\
\text { days }\end{array}$ & $\begin{array}{l}H J D_{0} \\
\text { days }\end{array}$ & $\begin{array}{c}V \\
\text { max light }\end{array}$ & $\begin{array}{c}\log \left(T_{\text {eff }}\right)^{\mathrm{a}} \\
(\mathrm{K})\end{array}$ & $\begin{array}{c}\left.F W H M_{\text {unsat }}\right\rangle \\
\mathrm{km} \mathrm{s}^{-1}\end{array}$ & $\begin{array}{c}\sigma\left(F W H M_{\text {unsat }}\right) \\
\mathrm{km} \mathrm{s}^{-1}\end{array}$ & $\begin{array}{c}\left.V_{\text {macrot }}\right\rangle \\
\mathrm{km} \mathrm{s}^{-1}\end{array}$ & $\begin{array}{c}\sigma\left(V_{\text {macrot }}\right) \\
\mathrm{km} \mathrm{s}^{-1}\end{array}$ \\
\hline as123811-1500 & -1.39 & 0.329045 & 1884.454 & 11.4 & 3.851 & 13.2 & 0.18 & 7.3 & 0.12 \\
\hline as132448-0658 & -2.04 & 0.343237 & 1900.300 & 11.4 & 3.851 & 16.3 & 0.12 & 9.3 & 0.07 \\
\hline as143322-0418 & -1.48 & 0.249632 & 1907.348 & 10.6 & 3.851 & 4.8 & 0.25 & 1.3 & 0.32 \\
\hline as211932-1507 & -1.50 & 0.273458 & 1873.307 & 11.1 & 3.851 & 9.9 & $\ldots$ & 5.0 & $\ldots$ \\
\hline \multicolumn{10}{|c|}{ MP RRc Blazhko ${ }^{\mathrm{d}}$} \\
\hline as110522-2641 & -1.60 & 0.294457 & 4900.170 & 11.7 & 3.851 & 15.6 & $\ldots$ & 8.8 & $\cdots$ \\
\hline as162158+0244 & -1.83 & 0.323698 & 4900.435 & 12.6 & 3.851 & 25.1 & $\cdots$ & 12.1 & $\cdots$ \\
\hline as200431-5352 & -2.66 & 0.300241 & 4900.470 & 11.0 & 3.851 & 15.4 & 2.00 & 8.7 & 1.28 \\
\hline CS22191-029 & -2.70 & $\cdots$ & $\cdots$ & 14.1 & 3.778 & 14.99 & $\cdots$ & 8.42 & $\cdots$ \\
\hline CS22875-029 & -2.65 & $\ldots$ & $\ldots$ & 13.7 & 3.778 & 15.31 & $\ldots$ & 8.57 & $\ldots$ \\
\hline CS22878-121 & -2.37 & $\ldots$ & $\ldots$ & 14.0 & 3.736 & 10.32 & $\ldots$ & 5.71 & $\ldots$ \\
\hline CS22879-097 & -2.45 & $\ldots$ & $\ldots$ & 14.2 & 3.763 & 13.16 & $\ldots$ & 7.46 & $\ldots$ \\
\hline CS22879-103 & -2.10 & $\ldots$ & $\ldots$ & 14.3 & 3.762 & 14.80 & $\ldots$ & 8.27 & $\ldots$ \\
\hline CS22881-039 & -2.68 & $\ldots$ & $\ldots$ & 15.1 & 3.785 & 13.62 & $\ldots$ & 7.59 & $\cdots$ \\
\hline CS22882-001 & -2.48 & $\cdots$ & $\cdots$ & 14.8 & 3.775 & 15.21 & $\cdots$ & 8.45 & $\cdots$ \\
\hline CS22883-037 & -1.89 & $\ldots$ & $\ldots$ & 14.7 & 3.771 & 15.72 & $\ldots$ & 8.93 & $\cdots$ \\
\hline CS22886-043 & -2.15 & $\ldots$ & $\ldots$ & 14.7 & 3.778 & 15.24 & $\ldots$ & 8.47 & $\ldots$ \\
\hline CS22888-047 & -2.34 & $\ldots$ & $\ldots$ & 14.6 & 3.778 & 14.93 & $\ldots$ & 8.34 & $\ldots$ \\
\hline CS22945-056 & -2.90 & $\ldots$ & $\ldots$ & 14.1 & 3.763 & 14.93 & $\ldots$ & 8.40 & $\ldots$ \\
\hline CS22948-006 & -2.62 & $\cdots$ & $\cdots$ & 15.1 & 3.744 & 11.60 & $\cdots$ & 6.51 & $\cdots$ \\
\hline CS22951-077 & -2.42 & $\ldots$ & $\ldots$ & 13.6 & 3.728 & 10.27 & $\ldots$ & 5.66 & $\cdots$ \\
\hline CS22955-174 & -2.88 & $\ldots$ & $\ldots$ & 14.4 & 3.752 & 14.15 & $\ldots$ & 8.08 & $\ldots$ \\
\hline
\end{tabular}

${ }^{a}$ For RRab stars the $\log T_{\text {eff }}$ values are means over the pulsational cycles, as given by Skarka (2014). For RRc stars we have adopted a constant for $\log T_{\text {eff. }}$.

${ }^{b}$ We gathered du Pont echelle spectra for this star but did not include it in our model atmosphere analyses in previous papers.

${ }^{c}$ Nominal periods; see Table 3 and text.

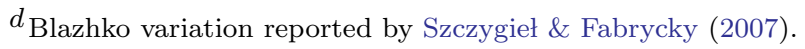


Table 2. Atomic Lines ${ }^{\mathrm{a}}$

\begin{tabular}{ccr}
\hline \hline$\lambda$ & Species & $\begin{array}{c}E W_{\odot}^{\mathrm{b}} \\
\AA\end{array}$ \\
\hline 4045.82 & Fe I & 1174 \\
4062.45 & Fe I & 98 \\
4063.60 & Fe I & 787 \\
4067.99 & Fe I & 133 \\
4071.75 & Fe I & 723 \\
4076.64 & Fe I & 127 \\
4077.72 & Sr II & 428 \\
4121.32 & Co I & 125 \\
4134.68 & Fe I & 129 \\
4143.42 & Fe I & 134 \\
\hline
\end{tabular}

$a_{\text {This table is available in its }}$ entirety in in the on-line article

$b_{\text {Equivalent widths from the }}$ Moore et al. (1966) Solar Atlas 
Table 3. Ephemerides for Blazhko Stars Used in Figure 10

\begin{tabular}{lccr}
\hline \hline \multicolumn{1}{c}{ Star } & HJD $_{0}$ & \multicolumn{1}{c}{$P$} & \multicolumn{1}{c}{$\delta \phi$} \\
\hline AM Vir & 1888.395 & 0.615085 & -0.010 \\
AS Vir & 3836.325 & 0.553400 & 0.000 \\
BS Aps & 3836.370 & 0.582560 & 0.015 \\
RV Cap & 5328.400 & 0.447748 & -0.010 \\
RV Cet & 5455.460 & 0.623421 & 0.025 \\
S Ara & 2137.338 & 0.451883 & -0.030 \\
UV Oct & 3836.795 & 0.542573 & 0.010 \\
V1645 Sgr & 3932.480 & 0.552910 & -0.010 \\
V Ind & 1873.466 & 0.479602 & -0.030 \\
V494 Sco & 5364.435 & 0.427266 & -0.045 \\
\hline
\end{tabular}


Table 4. Mean Values of $V_{\text {macrot }} / V_{\text {mic }}$ for MP RRab Stars

\begin{tabular}{cc}
\hline \hline$\phi$ & $\left\langle V_{\text {macrot }} / V_{\text {mic }}\right\rangle$ \\
\hline 0.025 & 4.37 \\
0.075 & 3.71 \\
0.125 & 3.11 \\
0.175 & 2.93 \\
0.225 & 2.55 \\
0.275 & 2.27 \\
0.325 & 1.91 \\
0.375 & 1.81 \\
0.425 & 2.08 \\
0.475 & 2.40 \\
0.525 & 2.69 \\
0.575 & 2.98 \\
0.625 & 3.28 \\
0.675 & 3.41 \\
0.725 & 3.23 \\
0.775 & 3.31 \\
0.825 & 3.41 \\
0.875 & 3.78 \\
0.925 & 4.05 \\
0.975 & 4.32 \\
1.025 & 4.37 \\
\hline
\end{tabular}


Table 5. $T_{\text {eff }}$ and $V_{\text {rot }} \sin (i)$ Values for RHB Stars in Two Previous Studies

\begin{tabular}{lcccc}
\hline \hline \multicolumn{1}{c}{ Source } & Star & $T_{\text {eff }}$ & $\log T_{\text {eff }}$ & $V_{\text {rot }} \sin (i)$ \\
\hline Behr (2003b) & HD 25532 & 5553 & 3.745 & 7.7 \\
Carney et al. (2008) & HD 25532 & 5320 & 3.726 & 4.8 \\
Difference & & 233 & 0.019 & 2.9 \\
& & & & \\
Behr (2003b) & HD 184266 & 5760 & 3.760 & 9.3 \\
Carney et al. (2008) & HD 184266 & 5490 & 3.740 & 5.0 \\
Difference & & 233 & 0.021 & 4.3 \\
& & & & \\
Mean Difference & & 252 & 0.020 & 3.6 \\
\hline
\end{tabular}


Table 6. Metallicity Data for Three RHB Samples

\begin{tabular}{lccc}
\hline \hline \multicolumn{1}{c}{ Source } & $\langle[\mathrm{Fe} / \mathrm{H}]\rangle$ & $\sigma$ & $f([\mathrm{Fe} / \mathrm{H}]>-1.7)$ \\
\hline This study & -2.59 & 0.27 & 0.00 \\
Carney et al. (2008) & -1.95 & 0.35 & 0.17 \\
Behr (2003b) & -1.53 & 0.36 & 0.80 \\
\hline
\end{tabular}

